\title{
How Do We Get Our Beliefs for Statistical Discrimination?
}

From a decision theoretical perspective, there is one major question that the concept of statistical discrimination raises: When is it rational to have a certain belief and use it for statistical discrimination and when not? By definition, the correctness of a statistical difference between two or more groups regarding some characteristic is not a requirement for statistical discrimination (LippertRasmussen, 2014). You also discriminate statistically if the difference and / or its relevance does not actually exist but you believe it to exist. But what defines the boundary between a rational and an irrational belief then?

On one hand, this is important so as to generally distinguish rational statistical discrimination from irrational statistical discrimination, meaning statistical discrimination that stems from irrational beliefs. On the other hand, from an empirically observational perspective, we need to know this boundary so as to be able to detect whether a decision is based on pure statistical discrimination or involves hidden taste-based discrimination. For example, an employer might not employ any women because he believes that the performance of women is statistically significantly worse than the one of men. If asked whether there is any proof for his statement, he might cite some article he read that came to this conclusion or his own experience: Of the last ten employees he had to fire, eight were female. For him, this is enough proof to believe that women perform worse than men. Now, are the employer's beliefs rational and, as a result, is not hiring any women merely applied statistical discrimination? Or is he a misogynist and thus a tastebased discriminator who wants to hide his resentment to women behind dubious

Electronic supplementary material The online version of this chapter (https://doi.org/10.1007/978-3-658-34569-3_4) contains supplementary material, which is available to authorized users.

D. Villiger, Dissecting Discrimination, Entscheidungs- und Organisationstheorie, https://doi.org/10.1007/978-3-658-34569-3_4 
beliefs that actually are irrational (and he knows that)? ${ }^{1}$ Or does the decisionmaker simply possess irrational beliefs, regardless whether he is a taste-based discriminator or not?

Let us look at what subjective expected utility theory says about this problem. Gilboa et al. (2012) write: "In modern economic thought, a decision maker who satisfies Savage (1954) axioms, and behaves as if they entertain a prior probability over a state space [the set of all scenarios], will be considered a rational decision maker under uncertainty, and may be viewed as having rational beliefs." (p. 12) Although the authors mention Savage's axioms this is also true in case of the assumptions that we needed for the Anscombe-Aumann representation theorem. Therefore, in subjective expected utility theory the rationality of beliefs is solely defined by internal consistency. In turn, which beliefs, or more precisely the subjective probabilities they result in, are internally consistent is defined by the assumptions needed for the Savage or Anscombe-Aumann representation theorem. ${ }^{2}$ This leads to the consequence that beliefs, which are highly implausible, can still be declared as rational as long as they are consistent with the other beliefs of the decision-maker. ${ }^{3}$

How does a rational decision-maker integrate new information into his current beliefs? In light of new data, a prior belief should be updated to a posterior belief according to Bayes' law which is as follows. Note that $e$ stands for new evidence.

$$
p\left(s_{i} \mid e\right)=\frac{p\left(e \mid s_{i}\right)}{p(e)} p\left(s_{i}\right)
$$

This means if new evidence $e$ is more likely in scenario $s_{i}$ than generally, the posterior probability of scenario $s_{i}$ increases. In contrast, given that new evidence $e$ is less likely in scenario $s_{i}$ than generally, the posterior probability of scenario $s_{i}$ decreases.

\footnotetext{
${ }^{1}$ This would imply that in the deciding situation he actually uses rational beliefs but has a taste for men and thus only hires men. When confronted by others, he tries to hide this taste for men behind irrational beliefs that he defends as being rational although he knows that they are not rational. Yet, it is also possible that he is not aware of the fact that he is a taste-based discriminator and really thinks that his different treatment is due to statistical discrimination (although it is actually due to taste-based discrimination).

2 This of course includes the three assumptions of probability theory defined by Kolmogorov (1933) that were presented in section 2.3.

3 There are economists that criticise such an understanding of rational beliefs (e.g. Gilboa et al., 2012).
} 
For example, an employer with agent-neutral preferences has a native and a foreign applicant with equal qualifications. There are three possible scenarios: $s_{1}$ $=$ "native applicant is more productive"; $s_{2}=$ "foreign applicant is more productive"; and $s_{3}=$ "both applicants are equally productive". Now, the employer believes that native workers are generally more productive than foreign workers. Therefore, he assigns $s_{1}$ a higher subjective probability than $s_{2}$ (e.g. $p_{1}=0.8$, $p_{2}=0.1$, and $\left.p_{3}=0.1\right)$ and consequently hires the native worker. Yet, after two weeks he fires him due to low productivity. Luckily, the foreign applicant is still looking for a job. The employer hires him and observes that he is much more productive than any native worker in the company. Because of this new evidence, he updates his prior belief that native workers are generally more productive than foreign workers and thereby also his subjective probabilities for $s_{1}, s_{2}$, and $s_{3}$. The result is some posterior belief as for example native and foreign workers are on average equally productive leading to posterior subjective probabilities such as $p_{1}=0.2, p_{2}=0.2$, and $p_{3}=0.6$.

We see that from a decision theoretical point of view, the actual content of a decision-maker's beliefs is irrelevant for whether his actions are rational or not. As long as the following two requirements are fulfilled, the decision-maker is a rational statistical discriminator: (1) His beliefs are internally consistent and thus his preference orderings satisfy the seven assumptions needed for the AnscombeAumann representation theorem. (2) He updates these beliefs or more precisely the subjective probabilities they result in by use of Bayes' law. In turn, these two requirements for rational beliefs lead us to the following consequence: While subjective expected utility theory determines how posterior beliefs should be formed, it does not offer a theory of prior belief generation (Gilboa et al., 2012).

There are two opinions in the literature on how a decision situation with no prior evidence that is linked to it should be handled (Kolmar, forthcoming). On one hand, objective Bayesianism says that a decision-maker should apply the principle of insufficient reason. This principle implies that given there are $n$ mutually exclusive and collectively exhaustive scenarios that are only distinguishable by their names, each scenario should have a probability equal to $1 / n$ (Jaynes, 1968). On the other hand, subjective Bayesianism states that valid priors solely have to fulfil the three assumptions of probability theory (cf. Kolmogorov, 1933). As a consequence, a decision-maker can freely choose his prior beliefs as long as they adhere to this requirement (de Finetti, 1937).

Now, in light of new information, a decision-maker's prior belief is of course of utter importance for the formation of his posterior belief. Let's take an example of Gilboa et al. (2012): "Consider a graduate student who believes that he is among the best economists in the world. Assume that he assigns probability 1 to this event, and that he takes decisions so as to maximize his expected utility with 
respect to these views. In the face of new evidence (failing prelims for example), he employs Bayes's rule to update his probability. But since he ascribes zero probability to the event that he is not a supremely gifted economist, his updated beliefs are that his professors are simply not sufficiently smart to recognize the depth and importance of his ideas." (p. 13) This behaviour is perfectly rational out of the perspective of decision theory if throughout the process the student satisfies all seven assumptions needed for subjective expected utility theory. Yet, from an intuitive perspective, we probably agree that the student needs to be treated as delusional.

We can think of a similar example regarding a group specific belief, such as a person who believes with probability 1 that men are more intelligent than women. In case of new disconfirming information, he would reinterpret it in a way that allows him to keep up his prior belief. Declaring such behaviour as rational is somewhat unsatisfying though. Therefore, in this chapter, we want to closer examine how we truly form and update our beliefs and thereby see whether groups and group memberships are relevant in these processes as well. In so doing, we make use of 19 biases, which are listed in table 4.1. ${ }^{4}$ First, we look whether humans might have inherent prior beliefs that are directly or indirectly linked to groups. Second, we consider whether we truly update our beliefs by use of Bayes' law. Third, we analyse society's role in belief generation and particularly preservation.

\subsection{Inherent Prior Beliefs}

The idea of this chapter is that there are beliefs which are not learned but inherently held by humans without the need of prior evidence. In turn, these inherent beliefs shine through in our biases. In section 2.3, we partitioned a decisionmaker's beliefs $b$ : $\beta_{\theta}$ comprises group unspecific beliefs and $\beta_{\mu}$ group specific beliefs (except $A$, which is a own category). These two partitions can now again be partitioned into inherent and learned beliefs, where $\gamma$ stands for inherent and $\lambda$ stands for learnt. ${ }^{5}$ This leads to four types of beliefs ( $A$ excluded): $\beta_{\theta^{\gamma}}, \beta_{\theta^{\lambda}}$, $\beta_{\mu^{\gamma}}$, and $\beta_{\mu^{\lambda}}$.

\footnotetext{
${ }^{4}$ Appendix B reveals how these 19 biases were chosen and appendix $\mathrm{C}$ introduces each of these 19 biases in more detail. Both appendices can be found in the electronic supplementary material.

5 The principle of insufficient reason could be seen as an inherent group unspecific belief that helps us to assign subjective probabilities in a decision situation where no prior evidence is available and scenarios appear symmetric.
} 
Table 4.1 The relevant biases for chapter 4

\begin{tabular}{l|l}
\hline \multicolumn{2}{c}{ Decision-Making, Belief, and Behavioural Biases } \\
heuristic & $\begin{array}{l}\text { The unconscious inference that high representativeness of an } \\
\text { object regarding a category automatically implies high probability } \\
\text { that the object also belongs to that category (Kahneman, 2011). }\end{array}$ \\
\hline Availability heuristic & $\begin{array}{l}\text { The unconscious inference that high availability of an incident or } \\
\text { characteristic implies high probability/frequency of these } \\
\text { (Kahneman, 2011). }\end{array}$ \\
\hline Illusory correlations & $\begin{array}{l}\text { Beliefs that incorrectly link a category with certain attributes or } \\
\text { another category (Meiser \& Hewstone, 2006). }\end{array}$ \\
\hline Omission bias & $\begin{array}{l}\text { People tend to judge harmful omissions as (morally) better than } \\
\text { equally harmful actions (Baron \& Ritov 2004). }\end{array}$ \\
\hline Negativity bias & $\begin{array}{l}\text { Humans have a tendency to weigh negative entities such as } \\
\text { personal traits, objects, or events more heavily than positive ones } \\
\text { (Rozin \& Royzman, 2001). }\end{array}$ \\
\hline Loss aversion & $\begin{array}{l}\text { The tendency that losses loom larger than same sized gains } \\
\text { (Kahneman, 2011). }\end{array}$ \\
\hline Status quo bias & $\begin{array}{l}\text { People tend to remain at the current state of affairs and prefer it to } \\
\text { possible changes (Kahneman et al., 1991). }\end{array}$ \\
\hline Confirmation bias & $\begin{array}{l}\text { The human tendency to seek or interpret evidence in ways that } \\
\text { are confirming existing beliefs, hypothesis, or expectations } \\
\text { (Nickerson, 1998). }\end{array}$ \\
\hline Backfire effect & $\begin{array}{l}\text { Disconfirming evidence might not lead to an adaption but } \\
\text { reinforcement of previous beliefs (Nyhan \& Reifler, 2010). }\end{array}$ \\
\hline $\begin{array}{l}\text { After a misinformation, which was initially presumed to be } \\
\text { correct, has been corrected it can still influence a person's belief } \\
\text { (Johnson \& Seifert, 1994). }\end{array}$ \\
\hline $\begin{array}{l}\text { The tendency to reject new evidence because it contradicts } \\
\text { established norms, beliefs, or paradigms (Mortell et al., 2013). }\end{array}$ \\
\hline Bonfluence
\end{tabular}


Table 4.1 (continued)

\begin{tabular}{l|l}
\hline \multicolumn{2}{c}{ Social Biases } \\
\hline Outgroup homogeneity bias & $\begin{array}{l}\text { The belief that outgroup members are all alike, whereas } \\
\text { ingroup members are diverse (Park \& Judd, 1990). }\end{array}$ \\
\hline Ultimate attribution error & $\begin{array}{l}\text { The phenomenon that people overemphasise situational } \\
\text { factors in case of negative behaviour of their own group but } \\
\text { personal factors in case of negative behaviour of other } \\
\text { groups. Moreover, positive acts tend to be attributed to } \\
\text { situational factors less when they are performed by an } \\
\text { ingroup member than when they are performed by an } \\
\text { outgroup member (Pettigrew, 1979). }\end{array}$ \\
\hline Linguistic intergroup bias & $\begin{array}{l}\text { The way people describe the behaviours of the ingroup and } \\
\text { outgroup varies in their level of abstraction. Positive ingroup } \\
\text { and negative outgroup behaviours tend to be described in } \\
\text { abstract terms. Negative descriptions of the ingroup and } \\
\text { positive descriptions of outgroups are prone to be made in } \\
\text { concrete terms (Maass et al., 1989). }\end{array}$ \\
\hline Illusion of truth effect & $\begin{array}{l}\text { Memory Errors and Biases } \\
\text { People are more likely to believe a statement they } \\
\text { previously heard than an unfamiliar one (Begg et al., 1992). }\end{array}$ \\
\hline Stereotypical bias & Stereotypes can distort our memory (Payne et al., 2004). \\
\hline Rosy retrospection & $\begin{array}{l}\text { The tendency to remember past events as having been more } \\
\text { positive than they actually were (Norman, 2009). }\end{array}$ \\
\hline $\begin{array}{l}\text { The propensity to perceive an event that has happened as } \\
\text { having been predictable even if it was not or very little } \\
\text { predictable (Wood, 1978). }\end{array}$ \\
\hline $\begin{array}{l}\text { When remembering past choices, people tend to attribute } \\
\text { positive features to chosen options and negative features to } \\
\text { rejected options (Henkel \& Mather, 2007). }\end{array}$ \\
\hline Hingortive bias
\end{tabular}

Given there truly are inherent prior beliefs, these must have emerged and prevailed in the course of evolution, which implies that there should be an evolutionary ultimate explanation for them. Therefore, this chapter has three goals: (1) if possible, bundle various biases that seem to be manifestations of the same inherent belief; (2) find an evolutionary ultimate explanation for the existence of the inherent belief; and (3) see whether these biases are universal so as to strengthen the argument that they truly are inherent and not learned. It has to be highlighted that particularly the last two goals are a rather speculative endeavour since in this area, research is often scarce. 
In the first part, we examine an inherent belief that is actually group unspecific, yet, can still affect group outcomes indirectly. Here, the following biases are of relevance: rosy retrospection, choice supportive bias, omission bias, loss aversion, negativity bias, and the status quo bias. In the second part, we concentrate on group specific inherent beliefs that derive from the outgroup homogeneity bias, the ultimate attribution error, and the linguistic intergroup bias.

\subsubsection{Prior Beliefs about Familiar and Unfamiliar Alternatives}

If we analyse table 4.1 , it seems like there is a superordinate cluster that inheres the following characteristics: Multiple biases make us wrongly anticipate the utility of familiar and / or unfamiliar alternatives. We can formulate this as follows: A choice set $F$ contains two alternatives, where $f_{1}$ is familiar and $f_{2}$ is unfamiliar to the decision-maker. This leads to three scenarios: $s_{1}=$ "familiar alternative is better", $s_{2}=$ "unfamiliar alternative is better", and $s_{3}=$ "both alternatives are equally good / bad". Of course, if $p_{1}$ is larger than $k_{2}$, the decision-maker chooses the familiar alternative or vice versa. However, the fact that he has hardly any information about the unfamiliar alternative $f_{2}$ complicates the formation of prior subjective probabilities.

Now, in such a situation, it seems that we systematically overestimate $p_{1}$ and thereby the subjective probability of $s_{1}$ due to the following biases. They do so in different ways. Rosy retrospection and the choice supportive bias make us overestimate the positivity of the past respectively past choices and thereby what we are familiar with (Norman, 2009; Henkel \& Mather, 2007). The omission bias makes us overestimate the expected utility of the status quo (the familiar option) because we judge harmful omissions as (morally) better than equally harmful actions (Baron \& Ritov 2004). Loss aversion and the negativity bias make us underestimate the expected utility of the unfamiliar alternative because we emphasis the dangers of the unfamiliar alternative and neglect its opportunities (Tversky \& Kahneman, 1991; Rozin \& Royzman, 2001). ${ }^{6}$ Finally, the status quo bias either makes us overestimate the expected utility of the status quo or underestimate that

\footnotetext{
${ }^{6}$ It is important to notice that this is only one side of loss aversion. The other side is that if our status quo is threatened, we rather defend it even though its expected value is lower and variance higher than those of the unfamiliar alternative. For example, there is a lottery with two options: (A) lose $\$ 1000$ or $\$ 0$ each with $50 \%$ chance; (B) lose $\$ 450$ for sure. Here, many would choose option A although its expected value is lower and variance higher (Kahneman, 2011). But they do so because option A provides the only chance to remain the status quo. This also demonstrates that loss aversion is not the same as risk aversion.
} 
of change (Kahneman et al., 1991). To summarise, these biases lead to a systematic distortion of our predictions: If confronted with change, we overestimate the expected utility that the familiar alternative provides while underestimating the expected utility of the unfamiliar alternative. As a consequence of this, our subjective probability of the scenario which comprises that the familiar alternative is better turns out larger than it should be. We call this the belief in the superiority of familiar alternatives.

Of course, this last paragraph could be a fallacy. How should we know that people truly systematically over- and underestimate the expected utility of familiar respectively unfamiliar alternatives and not simply have a preference for familiar alternatives (or are anxious about the unknown)? For example, let's assume that someone refuses to buy a computer and rather handles all administrational and informational matters analogue. The reason for that could be a status quo bias because of which he underestimates the expected utility of the new technology and / or overestimates the costs that are linked to this change. Yet, it could also be that he correctly anticipates the expected utility of buying a computer and still does not choose this alternative because maintaining the old-fashioned, familiar way to handle his matters simply provides him more expected utility. If this is the case, sticking to the status quo is not an expression of a bias but a preference.

The decisive question to solve this problem is as follows: How good are we at predicting future positive and negative affects? Given we anticipate positive and negative affects equally well (or badly), there should not be a systematic over- and underestimation of familiar respectively unfamiliar alternatives. However, given we tend to overestimate negative future affects, we do not simply have a preference for the familiar alternative but a belief in the superiority of familiar alternatives. Before we get to the explanation of this statement let us look at the evidence in the affective forecasting literature. First of all, there is a broad consensus that our affective forecasting abilities are limited (e.g. Buehler \& McFarland, 2001; Sanna \& Schwarz, 2004; Wilson \& Gilbert, 2003). We tend to overestimate the intensity and duration of our affective reactions in case of various focal events. This phenomenon is called the impact bias (Wilson \& Gilbert, 2003). Second, this impact bias displays a positive-negative asymmetry, meaning that it is much more pronounced for negative events compared to positive events (Buehler \& McFarland, 2001; Gilbert et al., 1998; Finkenauer et al., 2007). For example, Finkenauer et al. (2007) examined participants' ability to forecast their affect when they passed or failed their driving test. While affective forecasting differed from experienced affect in general, this was particularly true for negative affect. The authors summarise that "these findings closely replicate previous findings on 
the positive-negative asymmetry for the impact bias. In their forecasts, participants overestimate the intensity of their negative affect following the failure of an important exam much more than they overestimate their positive affect following the success of an important exam." (p. 1159)

The apparent positive-negative asymmetry of the impact bias strengthens our idea of a belief in the superiority of familiar alternatives. Since we are already experienced with the familiar alternative / the status quo, we are comparatively good at forecasting the affect that it produces. Contrary to that, in case of the unfamiliar alternative we overestimate the negative affect it might lead to. As a result, there are situations where we rather choose the familiar alternative / stick to the status quo than trying something new even though the unfamiliar alternative would actually have provided more expected utility. Yet, we did not realise that due to wrongly assigned subjective probabilities.

Now, are the above-mentioned biases linked to the impact bias and thereby display a positive-negative asymmetry? We begin with loss aversion. Loss aversion is usually explained via the asymmetrical impact of losses and gains, meaning that losses loom larger than same sized gains (Kahneman, 2011). Yet, most experiments that came to this inference either involved hypothetical decisions or did not measure the actual affective response after the decision was made and the outcome experienced. So, it is unclear whether loss aversion might actually stem from an affective forecasting error (at least partly). Kermer et al. (2006) investigated this question. In accordance with loss aversion, they found that participants predicted losses to have a greater emotional impact than gains of equal magnitude in a gambling task. Yet, when participants actually gambled, the impact of losses (and to a lesser degree also gains) was smaller than they predicted. In other words, the authors found an impact bias with a positive-negative asymmetry.

Regret is an essential element of the omission bias. This is because people seem to expect that bad effects of actions lead to greater regret than bad effects of omissions (Ritov \& Baron, 1995). As a consequence, given that a decisionmaker wants to avoid regret, he tends to prefer omissions to actions. Yet, as the affective forecasting literature shows, expected regret does not have to match with experienced regret, with the former tending to be larger than the latter (Gilbert et al., 2004). Now, the omission bias has often been connected with the decision not to vaccinate (Ritov \& Baron, 1990; Asch et al., 1994; Brown et al., 2010). Chapman and Coups (2006) examined anticipated and experienced regret regarding the decision to get a flu shot. They found that those who got the flu shot massively overestimated how much regret this decision would evoke. In contrast, those who did not get a flu shot showed no significant difference between anticipated and experienced regret. As a consequence, compared to omissions, people 
seem to overestimate the expected regret that the effects of an action might lead to. In turn, this implies that the omission bias is at least partly due to affective forecasting errors.

Unfortunately, there is no study that directly links the status quo bias with affective forecasting. Nevertheless, there are indications that affective forecasting could be relevant here as well. This is because loss aversion and regret avoidance are said to be important mechanisms behind the status quo bias (Anderson, 2003; Kahneman et al., 1991; Eidelman \& Crandall, 2012). So, if affective forecasting errors are relevant for them, they should also be relevant for the status quo bias. This implicates that "at least sometimes, the tendency to stick to the status quo results from affective forecasts rather than from affective experience". (Zamir, 2014, p. 271).

What about the negativity bias, rosy retrospection, and the choice-supportive bias? The negativity bias is connected with loss aversion because it involves that we weigh negative outcomes more heavily than positive outcomes (Hochman \& Yechiam, 2011). Due to that it is not far-fetched to assume the positive-negative asymmetry of the impact bias and the negativity bias are somehow intertwined. Within rosy retrospection, we can directly identify the impact bias. For example, Mitchell et al. (1997) showed that anticipation of holidays was generally more positive than actual experience, which is equivalent to the impact bias regarding positive events. So far, so good. Now, interestingly, in retrospection the holidays were perceived more positively than they actually had been in the moment of experience. A prominent explanation for this effect is that negative affect tends to fade faster than positive affect (Ritchie et al., 2015). Consequently, if people had to again choose a holiday trip, they would overestimate the positivity of those already chosen in the past which leads to an affective forecasting error. Finally, the choice-supportive bias has per se nothing to do with affective forecasting. Yet, as rosy retrospection, it might help to maintain an impact bias in case of already chosen options. The bias includes that " $[w]$ hen remembering past choices, people tend to attribute positive features to chosen options and negative features to rejected options" (Henkel \& Mather, 2007, p. 163). This is even true if they misremember or got misled concerning their actually chosen option. Therefore, compared to rejected options, people seem to overestimate the positivity of chosen options. In turn, this would lead to affective forecasting errors if a choice set, among others, also includes a formerly chosen option. 
We see that the analysis of this chapter's biases regarding their connection to affective forecasting indicates that they at least sometimes do influence the formation of subjective probabilities. At this, they seem to promote a belief in the superiority of familiar alternatives. Now, out of an evolutionary perspective, it is on first sight questionable why such an inherent belief should be adaptive. It could be argued that sticking to the familiar alternative should only make sense if the expected consequences of the familiar alternative are better than those of the unfamiliar one. Thus, there should be no favouritism for the familiar alternative in and of itself. For example, let us assume that an environment has three kinds of berries (berry 1,2, and 3) and two populations (group A and group B). While group A does not belief in the superiority of familiar alternatives, group B does. Now, both groups try all three berries and realise that berry 2 and 3 are inedible, whereas berry 1 is nutritious and well-tolerated. As a consequence, both groups exclusively eat berry 1 . After a few generations, the groups still only eat berry 1 although their members have never tried the other berries. All of a sudden, a new berry (berry 4) appears. Group A tries this new berry and realises that it is even better than berry 1 . Thus, they start to mainly eat berry 4 and in so doing increase their fitness. Meanwhile, group B does not try this new berry and simply sticks to the status quo. Since fitness of the members of group A is higher than that of the members of group B, the former should supersede the latter as time goes by.

Of course, this example is very simplified. However, precisely food is an area where familiarity is crucial to us. The popular proverb "some people won't eat anything they've never seen before" demonstrates this. Indeed, our eating habits are highly correlated with our culture. While eating cats, dogs, or guinea pigs is unthinkable in Europe, in other countries it is a common dish. However, even within their own food culture, most people order the same food in the same restaurants most of the time (Hall, 1992). Why are we not more adventurous? Rozin (1990) argues that our scepticism in new food functions as a defence system against potentially dangerous substances. Thus, unfamiliar food is rejected because we consciously or unconsciously fear to get poisoned and endanger our health. ${ }^{7}$ The finding that the degree of perceived dangerousness of food predicts the subsequent willingness to try unfamiliar food supports this hypothesis (Pliner et al., 1993; Lähteenmäki \& Arvola, 2001).

\footnotetext{
${ }^{7}$ Of course, if food is scarce in general, you should not be too picky because otherwise you die due to malnutrition. Thus, this explanation is based on the underlying environmental assumption that food was not scarce but rather abundant. We will discuss the importance of these environmental assumptions in a moment.
} 
Can we expand this explanation for why we favour familiar food on familiar alternatives in general? Let us begin with the theoretical concept that lies underneath that explanation: error management theory (Haselton \& Buss, 2000; Haselton \& Nettle, 2006; Johnson et al., 2013). "Error management theory ... applies the principles of signal detection theory (Green \& Swets, 1966) to judgment tasks in order to make predictions about evolved cognitive design." (Haselton et al., 2015, p. 972) The idea is as follows: The goal of our cognitive mechanisms is not per se accuracy (e.g. Fodor, 2001) but adaptiveness (e.g. Tooby \& Cosmides, 1990). While these two sometimes go together, they do not have to. There are two reasons why this is true. First, our cognitive mechanisms are seldomly perfectly accurate. Normally, real-world judgments involve an irreducible amount of uncertainty. As a consequence, our cognitive mechanisms produce errors. Second, there are two kinds of errors: false negative (failing to take an action that would have been better to take) and false positive (taking an action that would have been better not to take). For example, if you do not eat a certain berry because you think that it is not edible, yet, it actually is, we have a case of false negative. In contrast, if you eat a certain berry because you think that it is edible, yet, it actually is not, we have a case of false positive. Now, given the costs of these two errors are exactly the same, ceteris paribus, the more accurate you are the higher is your fitness. However, this is no longer true if the costs of false negative and false positive are asymmetric. Let us illustrate this by means of a fire detector. Here, the two possible errors are as follows: (1) The fire detector bells even though there is no fire (false positive). (2) The fire detector does not bell even though there is a fire (false negative). Of course, here, the costs of false negative typically are much higher than those of false positive. Thus, a fire detector is not designed to be as accurate as possible and thereby minimise the overall error rate but to detect as many fires as possible and thereby minimise false negatives: You rather have an alarm system that occasionally bells even though there is no fire than an alarm system that occasionally does not bell although there is a fire, yet, overall is more accurate (Haselton \& Nettle, 2006). ${ }^{8}$

\footnotetext{
${ }^{8}$ Minimise false negative errors does not automatically mean that there should be none of these errors. If this were the case, the alarm would simply have to bell all the time. Yet, then, the fire detector becomes obsolete. So, there is a trade-off between minimising false negative errors and minimising overall errors, which has to be considered when designing a fire detector.
} 
If we apply these considerations on the evolution of cognitive mechanisms, we realise that they are not designed to minimise our total error rate but the net effect of error on fitness. As Haselton et al. (2016) write: "Where one error is consistently more damaging to fitness than the other, EMT [error management theory] predicts that a bias toward making the less costly error will evolve- this is because it is better to make more errors overall as long as they are relatively cheap." (p. 973) That is exactly what we might observe in case of food, particularly in regard to children (Cashdan, 1998; Dovey, 2010). Provided that there is abundant food, trying unfamiliar food that actually is poisonous is costlier than not trying some unfamiliar food that actually is edible. As a result, we favour familiar food so as to minimise false positive. Indeed, such a cognitive mechanism leads to more errors than one whose purpose is to be as accurate as possible. Yet, they are relatively inexpensive and therefore better than occasional disastrous errors.

Now, the emergence of an inherent belief in the superiority of familiar alternatives needs the following circumstances: The costs of the possible errors regarding the decision of whether a familiar or an unfamiliar alternative should be chosen are at least sometimes asymmetric. And given they are asymmetric, the costs of choosing an unfamiliar alternative although it provides less expected utility are generally higher than the costs of not choosing an unfamiliar alternative although it would provide more expected utility. But could such a belief truly evolve?

In order to answer this question, we have to ask a follow-up question, namely, how much openness for unfamiliar options was most adaptive in our past environment. As mentioned several times, the costs of false negative and false positive depend on the environment. For instance, if food is scarce, the costs of not trying unfamiliar food although it is edible can be higher than those of trying unfamiliar food although it is not edible. This is true if familiar food alone is not sufficient to guarantee survival either way. Thus, if you never try unfamiliar food because you are not open to unfamiliar alternatives, you die from malnutrition for sure. In contrast, if you try unfamiliar food because you are open to unfamiliar alternatives, you might die from food poisoning but maybe also find a new edible food source that enables your survival. In other words, if the bird in the hand is not enough either way, you better go for the two in the bush.

Importantly, even if the environment led to cost asymmetry that either promoted a bias towards familiar or unfamiliar alternatives, this does not imply that people would therefore always choose the respective alternative. Let's take the belief in the superiority of familiar alternatives. It says that we overestimate the 
subjective probability that the familiar alternative is better. Yet, despite this overestimation, our subjective probability that the unfamiliar alternative is better might still be higher, which is why we then choose the unfamiliar alternative.

Now, given we truly have an inherent belief in the superiority of familiar alternatives, the environment in which humans evolved had to be stable enough in order that false negative became costlier than false positive. However, since instability can have countless manifestations it is hardly possible to precisely determine how stable the environment needed to be in order that false positive was costlier than false negative. The only thing we know for sure is that our environment was not completely stable (e.g. Martrat et al., 2004). As a result, the question of whether our environment truly led to such a cost asymmetry is a bit pointless. So, let us rather examine a consequence that would stem from an inherent belief in the superiority of familiar: Given such a belief evolved during the course of evolution, it should be culture invariant.

Wang et al. (2017) examined loss aversion across 53 countries. They used the questionnaire of Hofstede (2001) on cultural dimensions so as to measure cultural differences. First of all, the results revealed that loss aversion existed in all cultures. However, there are substantial differences. Participants of cultures that score low in individualism, power distance, and masculinity also display a lower degree of loss aversion. ${ }^{9}$ Moreover, higher uncertainty avoidance led to more loss aversion, yet, less significantly than the other three dimensions. So, while loss aversion could be found in all examined cultures, its precise magnitude is culture-bound.

Concerning the status quo bias, there is no such cross-cultural analysis. Yet, Fernandez and Rodrik (1991), who studied the status quo bias regarding policy reforms, provide evidence that people of non-Western countries experience such a bias as well. They write: "A striking paradox, particularly in developing countries, is that while trade reform typically turns out to be a boon to large segments of the private sector, these same groups are rarely enthusiastic about reform early on. This is a pattern observed in Taiwan and South Korea (early 1960's), Chile (1970's), and Turkey (1980's) ... In all three cases, reform was imposed by authoritarian regimes and against the wishes of business, even though business emerged as the staunchest defender of outward orientation once the policies were in place." (p. 1147)

\footnotetext{
${ }^{9}$ High power distance means that people accept that power is distributed unequally. High masculinity means that self-assertion, competition, and success are crucial (and for example not caring, which would be feminine).
} 
What about the other biases? There is no cross-cultural study regarding the omission bias. However, there are Asian studies that examine the omission bias. For example, the sample of Chung et al. (2014) consisted of Korean students. As in studies with Western subjects, the authors also found an omission bias, yet, only if participants had a prevention focus, meaning sensitivity to negative outcomes and losses. ${ }^{10}$ This makes sense because only a prevention focus suggests higher costs of false positive than false negative and therefore sticking to the familiar alternative (which is doing nothing). ${ }^{11}$ Nonetheless, there is at least one culture that does not show an omission bias. Abarbanell and Hauser (2009) investigated a small-scale, agrarian Mayan population and found that subjects did not judge omissions causing harm as better than respective actions. ${ }^{12}$ Thus, the omission bias could have a culture component. Yet, it might also be the very culture of this small-scale, agrarian Mayan population that disperses the omission bias. Concerning the negativity bias, there is no cross-cultural study, however, studies that were conducted in China or Japan also report a negativity bias (e.g. Huang \& Luo, 2006; Ito et al., 2017). Unfortunately, no cross-cultural or non-Western studies could be found for the choice supportive bias and rosy retrospection. To summarise, the belief in the superiority of familiar alternatives seems not to be culture-bound, which maintains the hypothesis that it is inherent.

How does this inherent belief affect groups? Let's say a choice set has two alternatives with the same characteristics $i$ but providers of different groups. One provider is identified as being part of $\mathcal{M}_{1}$ and the other as being part of $\mathcal{M}_{2}$, so $A=\left\{\mathcal{M}_{1}, \mathcal{M}_{2}\right\}$. This leads to the following choice set: $F=\left\{f_{i}^{\mathcal{M}_{1}}, f_{i}^{\mathcal{M}_{2}}\right\}$. We assume that there are three scenarios $\left(S=\left\{s_{1}, s_{2}, s_{3}\right\}\right): s_{1}=$ "provider of $\mathcal{M}_{1}$ is better", $s_{2}=$ "provider of $\mathcal{M}_{2}$ is better", and $s_{3}=$ "the two providers are equally good / bad". The scenarios subjective probabilities are a function of $\beta_{\theta^{\gamma}}, \beta_{\theta^{\lambda}}$, $\beta_{\mu^{\gamma}}, \beta_{\mu^{\lambda}}$, and $A$. Now, there are three further assumptions. (1) Regarding $\mathcal{M}_{1}$, the decision-maker has some / many group specific beliefs, including that $\mathcal{M}_{1}$ is familiar. (2) Regarding $\mathcal{M}_{2}$, the decision-maker has few group specific beliefs, including that $\mathcal{M}_{2}$ is unfamiliar. (3) Given the decision-maker cannot retrieve $\beta_{\theta^{r}}$ that contains the inherent belief in the superiority of familiar alternatives, he is indifferent between the two alternatives.

\footnotetext{
${ }^{10}$ In contrast, the promotion focus implies sensitivity to positive outcomes and gains.

${ }^{11}$ Unfortunately, the authors do not indicate how many participants had a prevention and how many a promotion focus.

12 Interestingly, the authors found an omission bias in the less rural and more educated Mayan comparison group.
} 


$$
\begin{aligned}
\forall f_{i}^{\mathcal{M}_{1}}, f_{i}^{\mathcal{M}_{2}} \in F & : \sum_{i=1}^{3} q_{i}\left(\beta_{\theta^{\lambda}}, \beta_{\mu^{\gamma}}, \beta_{\mu^{\lambda}}, A\right) u\left(f_{i}^{\mathcal{M}_{1}}\left(s_{i}\right)\right) \\
& =\sum_{i=1}^{3} q_{i}\left(\beta_{\theta^{\lambda}}, \beta_{\mu^{\gamma}}, \beta_{\mu^{\lambda}}, A\right) u\left(f_{i}^{\mathcal{M}_{2}}\left(s_{i}\right)\right)
\end{aligned}
$$

The third assumption is due to the fact that the decision-maker has hardly any information about the provider of $\mathcal{M}_{2}$, which is why his group specific beliefs are insufficient so as to properly assess whether $s_{1}$ or $s_{2}$ is more likely. ${ }^{13}$ However, if he can retrieve $\beta_{\theta \gamma}$, he can make use of inherent group unspecific beliefs and thereby the belief in the superiority of familiar alternatives. In this example, the familiar alternative self-evidently is the one that the person of the familiar group provides. This leads to a subjective probability distribution on $S$ where $p_{1}$ is larger than $p_{2}$.

$$
\begin{aligned}
\forall f_{i^{*}}^{\mathcal{M}_{1}}, f_{i^{*}}^{\mathcal{M}_{2}} \in F & : \sum_{i=1}^{3} q_{i}\left(\beta_{\theta^{\gamma}}, \beta_{\theta^{\lambda}}, \beta_{\mu^{\gamma}}, \beta_{\mu^{\lambda}}, A\right) u\left(f_{i^{*}}^{\mathcal{M}_{1}}\left(s_{i}\right)\right) \\
& >\sum_{i=1}^{3} q_{i}\left(\beta_{\theta^{\gamma}}, \beta_{\theta^{\lambda}}, \beta_{\mu^{\gamma}}, \beta_{\mu^{\lambda}}, A\right) u\left(f_{i^{*}}^{\mathcal{M}_{2}}\left(s_{i}\right)\right)
\end{aligned}
$$

It is important to distinguish the belief in the superiority of familiar alternatives from anxiety about the unknown that we introduced in section 3.3.3. The belief in the superiority of familiar alternatives is restricted to the formation of a subjective probability distribution. In contrast, anxiety about the unknown is equivalent to a preference for familiar alternatives. Here, even in a situation of decision-making under certainty, an alternative with characteristics $i$ gives more utility if it is provided by someone from a familiar compared to an unfamiliar group. In formal terms, where $\mathcal{M}_{\text {fam }}$ stands for the familiar and $\mathcal{M}_{\text {unf }}$ for the unfamiliar group, there is a sufficient case of anxiety about the unknown if:

$$
\exists x_{i}^{\mathcal{M}_{\text {fam }}}, x_{i}^{\mathcal{M}_{\text {unf }}} \in X: u\left(x_{i}^{\mathcal{M}_{\text {fam }}}\right)>u\left(x_{i}^{\mathcal{M}_{\text {unf }}}\right)
$$

\footnotetext{
${ }^{13}$ In certain situations, group specific beliefs about the familiar group might be sufficient so as to assess whether $s_{1}$ or $s_{2}$ is more likely. For example, if you want to go for dinner and have two alternatives whereby the familiar provider is a world-famous cook, it is probable that her meal is better than that of the unfamiliar cook. Of course, the opposite case is also possible given the familiar cock is known to be extraordinarily bad.
} 
Yet, despite this crucial difference between anxiety about the unknown and the belief in the superiority of familiar alternatives, the former's ultimate explanation might be provided by error management theory too. When meeting a stranger, you do not know whether she is friendly or hostile. Given the costs of assuming that the stranger is friendly although she actually is hostile (false positive) are higher than vice versa (false negative), it can be adaptive to develop a preference for familiar providers (cf. Haselton \& Nettle, 2006). Moreover, as previously mentioned in section 3.3.3, through intergroup contact the unfamiliar provider can become a familiar provider as well. This then dissolves the difference between the expected utility of the two alternatives that the belief in the superiority of familiar alternatives produced since there is no unfamiliar alternative left.

To summarise this chapter, in a decision situation where there is a familiar alternative and an unfamiliar alternative, people have an inherent belief in the superiority of the familiar alternative. Error management theory provides an ultimate explanation for this belief. If the costs of false negative and false positive errors are asymmetric, biased cognitive mechanisms should evolve. Our biases suggest costlier false positive errors. In turn, this suggests a rather stable environment. Yet, it is unclear what that exactly means. We only know that our environment was not perfectly stable. Finally, our cross-cultural analysis mainly revealed that favouring familiar alternatives is not limited to Western culture. Thus, we can maintain the hypothesis that the belief in the superiority of familiar alternatives has an evolutionary origin and thus is inherent.

\subsubsection{Prior Beliefs about the Ingroup and Outgroup}

In this chapter we discuss three biases that could be described as inherent prior beliefs about the ingroup and the outgroup: the outgroup homogeneity bias, the ultimate attribution error, and the linguistic intergroup bias. In so doing, we have to keep in mind that since these beliefs concern the ingroup and outgroup their exact manifestation is intertwined with the holder's social identity. ${ }^{14}$ Unfortunately, there is no literature about the evolution of these three biases. So, while reading this chapter, it has to be kept in mind that the following explanations are hypothesises that need further proof. Notwithstanding this limitation, the sole fact that groups have such conflicting beliefs about each other suggests that they could not have been formed in an exclusive objective Bayesian way.

\footnotetext{
${ }^{14}$ Appendix D which can be found in the electronic supplementary material reveals the interaction between social identity and these three biases in more detail.
} 
Let us begin with the outgroup homogeneity bias. This bias involves the belief that outgroup members are all alike, whereas ingroup members are diverse (Linville et al. 1989; Park \& Judd, 1990). Kite and Whitley (2016) mainly mention two lines of explanation for it. (1) Since we have more contact with the ingroup than with the outgroup, we also have more knowledge about the ingroup, including its diversity. (2) While outgroup members are primarily perceived through a group perspective, ingroup members are also perceived through an individual perspective (individuals compare themselves with fellow ingroup members). So, in case of outgroup members, mainly group membership is salient. Since all outgroup members of one group self-evidently have the same group membership, they seem rather homogenous. In case of ingroup members, both group membership and individual characteristics are salient. As a result, the ingroup appears more heterogenous than the outgroup. So, the outgroup homogeneity bias might not be an adaptation in and of itself but the product of a lack of knowledge and the unnecessity to further differentiate between outgroup members. This suggests that the belief that outgroup members are all alike, whereas ingroup members are diverse is actually learned and not inherent.

But the outgroup homogeneity bias could also be explained via an evolutionary approach because in intergroup conflicts, perceiving the outgroup as homogenous can also be fitness enhancing. Normally, in such a situation, there is a clear line: We are the good ones and our enemies are the bad ones (Brewer, 1999). So, all outgroup members are viewed as homogenously evil, which decreases empathy with them, up to the point of dehumanisation (Haslam, 2006; Shilo et al., 2018), and thereby facilitates the victory over them. ${ }^{15}$ In his book "All Quiet on the Western Front", Erich Maria Remarque (1975) impressively describes a scene, where a soldier loses his outgroup homogeneity and thereby the thinking that all enemies are evil. It happens when he deadly wounds an enemy in a ditch and has to accompany his slow death because it is too dangerous to leave the ditch. He says to the dead enemy soldier: "Comrade, I did not want to kill you. If you jumped in here again, I would not do it, if you would be sensible too. But you were only an idea to me before, an abstraction that lived in my mind and called forth its appropriate response. It was that abstraction I stabbed. But now, for the first time, I see you are a man like me. I thought of your hand-grenades, of your bayonet, of your rifle; now I see your wife and your face and our fellowship.

15 In line with that there is a close relationship between perceived outgroup homogeneity and the endorsement of outgroup stereotypes (Hewstone \& Hamberger, 2000; Park \& Hastie, 1987; Ryan et al., 1996). So, if a stereotype says that the outgroup is evil and someone perceives the outgroup in a homogenous way, she is likely to extensively endorse that stereotype of an evil outgroup. 
Forgive me, comrade. We always see it too late. Why do they never tell us that you are poor devils like us, that your mothers are just as anxious as ours, and that we have the same fear of death, and the same dying and the same agonyForgive me, comrade; how could you be my enemy? If we threw away these rifles and this uniform you could be my brother just like Kat and Albert. Take twenty years of my life, comrade, and stand up-take more, for I do not know what I can even attempt to do with it now." (p. 100) So, by realising that his enemies are humans just like himself and not homogeneously evil, he loses the willingness to kill them. Yet, this willingness is decisive in order to win intergroup conflicts and consequently in order that one's own group prevails.

The last two paragraphs provided two possible explanations for the outgroup homogeneity bias that either followed a learning or an evolutionary approach. Yet, the outgroup homogeneity bias actually depends on group status (which is connected to whether the group is part of the minority or majority) and strength of ingroup identification (Simon \& Brown, 1987; Lorenzi-Cioldi, 1998; de Cremer, 2001). ${ }^{16}$ Low status / minority groups are less likely to display an outgroup homogeneity bias. In fact, they even tend to perceive the ingroup as more homogenous than the outgroup. Additionally, the more a person identifies with her ingroup, the likelier she displays an ingroup homogeneity bias.

Let us first discuss the second phenomenon. Group identification leads to depersonalisation, meaning the individual adopts the identity and interests of the group (Brewer, 1999). Consequently, the more a person identifies with a group, the more that person defines herself in terms of the group. Due to this, when looking at fellow ingroup members, mainly group membership is salient, leading to the impression of a homogenous group. ${ }^{17}$ This is compatible with the idea that people learn the outgroup homogeneity bias. But then again, this whole process is advantageous in case of intergroup conflicts. This is because individuals who adopt the interests of their group and thoroughly follow the norm "one for one and one for all" increase group fitness and thus prevail, given there is a group selection mechanism. In line with that intergroup conflict elevates group identification (cf. Haidt, 2012) and therefore triggers this process that ultimately leads to higher group fitness, which in turn raises the chances that the group prevails. So, this appearance of an ingroup homogeneity bias could also have an evolutionary origin.

\footnotetext{
${ }^{16}$ In fact, the order of comparison is of importance as well (Bartsch \& Judd, 1993; Castano \& Yzerbyt, 1998). However, we neglect this here.

${ }^{17}$ As we said before, this is what happens anyway in case of the outgroup.
} 
To continue, there is evidence that minority group members may perceive their social identity more positively if they regard their ingroup as homogenous. The reason for this is that ingroup homogeneity is positively linked to ingroup solidarity (Lee \& Ottati, 1995; Simon \& Mummendey, 1990; Simon \& Pettigrew, 1990; Doosje et al., 1995). Behind this proximate explanation, we find the same ultimate explanation given in the last paragraph. Minority groups do not suffer an outgroup homogeneity bias but an ingroup homogeneity bias because that increases their group fitness. Ultimately, this is useful in order to compete against the majority group. Beside this evolutionary explanation, there is also a learning explanation for why minority groups perceive themselves as less heterogenous. Societies are usually dominated by majority / high-status groups (Sidanius \& Pratto, 2001). Consequently, they have a stronger impact on the determination of cultural beliefs. Now, due to the outgroup homogeneity bias, these groups spread the belief that minority / low-status groups are more homogenous. In turn, minority / low-status groups internalise this culturally dominant belief and start perceiving themselves as more homogenous than the outgroup.

So, the specifications of the outgroup homogeneity bias can be explained via both an evolutionary and a learning approach. Which one is more likely to be true? A meta-analysis of Boldry et al. (2007) provides an indication. First of all, they found a small but reliable tendency to perceive the outgroup more homogeneous than the ingroup in the 173 independent samples they examined. Secondly, and more importantly, this tendency could not be found in case of minimal groups. This supports the learning hypothesis out of the following reason: In a minimal group setting, it is not possible to acquire any beliefs about the ingroup and outgroup. Thus, if the belief that a certain outgroup is more homogenous than the ingroup is learned, we should not find it in case of minimal groups. Otherwise, if the belief were inherent, we should have also found it when we have not yet learned any beliefs about the ingroup and outgroup since the inherent belief can always be retrieved.

Now, given the outgroup homogeneity bias is learned, we might find cultural differences in its appearance. While there are no proper cross-cultural studies about the outgroup homogeneity bias, two papers give us a hint about its universality. Shilo et al. (2018) examined the outgroup homogeneity bias in both Israeli and German children as well as adults. The results revealed no cultural differences, yet, both cultures are also characterised by Western values. Lee and Ottati (1993) studied the outgroup homogeneity bias of Chinese and American participants, whereby the respective other group provided the outgroup. They found that in both cases, Americans were described as more heterogenous than the Chinese. There are two explanations for this finding: (1) Americans truly are a lot more 
divers than the Chinese because America is much more multi-cultural and multiethnical than China. (2) In Chinese culture, being homogenous has a positive value. In contrast, Americans positively value heterogeneity. Therefore, while the Chinese rather describe themselves as homogenous, Americans rather perceive themselves as heterogenous. Unfortunately, there is no study that analysed two groups that both have a Chinese background (and more or less the same objective homogeneity). Here, it would be interesting whether the positive value of homogeneity eliminates the outgroup homogeneity bias and might even provoke an ingroup homogeneity bias. Nevertheless, we see that culture plays a role in the display of the bias. This finding is compatible with the idea that the bias did not develop in the course of evolution. In conclusion, although the origin of the outgroup homogeneity bias and its different manifestations is still unclear, the evidence for the learning hypothesis is more convincing than that of the evolutionary hypothesis.

We continue with the other two biases. The ultimate attribution error and the linguistic intergroup bias have a lot in common. Both describe the phenomenon that we attribute and describe positive and negative ingroup behaviour in a more flattering / favourable (and thereby self-serving) way than positive and negative outgroup behaviour (Pettigrew, 1979; Maass et al., 1989). The two biases can also be seen as beliefs: While positive ingroup behaviour is due to the ingroup's skills and negative ingroup behaviour is accidental, it is precisely vice versa in case of the outgroup. Now, both the ultimate attribution error and the linguistic intergroup bias get stronger the more an individual identifies with her ingroup or might even only appear if there is strong group identification. ${ }^{18}$ This is why we assume that they have the same underlying ultimate explanation.

Let us begin with the psychological effect of the ultimate attribution error and the linguistic intergroup bias. Through misattribution and biased description, the two biases lead to a more positive social identity than a situation actually yields. From this perspective it also becomes obvious why they interact with group identification. The more a person identifies with a group, the keener she is in attaining a positive social identity by means of the group she identifies with. This is due to the fact that this group substantially defines her self-identity. Thus, the two biases could be explained through cognitive dissonance theory (Festinger, 1957): (1) I want a positive social identity. (2) A situation either attacks my social identity

\footnotetext{
${ }^{18}$ Admittedly, the results regarding the linguistic intergroup bias are in fact a bit more complex and might also depend on group status and other factors. Moreover, the interaction between the linguistic intergroup bias and group identification is not as straightforward as in case of the ultimate attribution error. Nevertheless, in this chapter we only consider the positive correlation between group identification and the two biases.
} 
(the ingroup does something bad in their own responsibility or the outgroup does something good in their own responsibility) or does not allow to improve it (the ingroup does something good out of luck or the outgroup does something bad out of bad luck). (3) In order to still maintain or improve my social identity, I misattribute the situation and describe it in a biased way.

Yet, this simply shifts the problem because now we have to ask what is the ultimate explanation of cognitive dissonance? In fact, there is hardly any research about that. For example, Perlovsky (2013) writes: "Why have researchers of CD [cognitive dissonance] theory, "the most influential and extensively studied theory in social psychology" not noticed this contradiction between its fundamental premise and the fact of human evolution?" (p. 2)

Might the two biases be advantageous in intergroup conflicts because they increase group fitness? For example, it could be argued that a more positive social identity facilitates depersonalisation and, in this way, ultimately leads to higher group fitness. However, in case of group conflicts, the two biases can also be disadvantageous. Let us assume that a group loses a conflict with another group. Attributing one's loss and the other's victory to situational and not group factors will not help to win the next conflict. In contrast, such a self-deceiving attribution probably results in another loss. Thus, a realistic assessment of the situation could be better for the survival of the group even though it leads to a less positive social identity. In the end, whether the biases are beneficial for a group depends on how strong each of these two effects are. Unfortunately, there are no studies that examine this topic. So, the ultimate explanation for the linguistic intergroup bias and the ultimate attribution error is still very unclear and further research is needed in order to get a proper hypothesis.

At least, there is evidence for the biases' universality. There are two studies that were conducted in Non-Western societies. The experiment of Khan et al. (2008) had Indians and Pakistanis as participants. In turn, Chan (2017) let Chinese (more precisely Hong Kongese) subjects fill out his questionnaires. While Khan et al. (2008) confirmed the existence of the ultimate attribution error, Chan (2017) did so in the case of the linguistic intergroup bias. Moreover, it seems unlikely that people individually learn the ultimate attribution error and the linguistic intergroup bias. This is because it is difficult to explain how groups that consider the same evidence systematically and universally come to totally different conclusions (e.g. the achievement was out of luck vs. the achievement was out of 
skill). ${ }^{19}$ Ultimately, on an individual level, there is ample evidence that people are overconfident / overoptimistic regarding themselves (Svenson, 1981; Brown 1986; Campbell, 1986; Hagerty, 2003; Sedikides et al., 2003). For example, Svenson (1981) asked car drivers from the US and Sweden how well and safe they think they drive compared to the other participants in the study. $93 \%(88 \%)$ of the US sample and $69 \%$ (77\%) of the Swedish sample believed themselves to be more skilful (safer) drivers than the median driver of their sample. Such beliefs could hardly be obtained in an objective Bayesian way, which suggests inherent overconfidence (Haselton \& Nettle, 2006). And this inherent overconfidence probably not only affects individual assessments but also group assessments, whereby the biased way we attribute ingroup and outgroup behaviour is one of the mechanisms that generates and helps us to uphold our overconfidence. As a consequence, we can maintain the hypothesis that the ultimate attribution error and the linguistic intergroup bias are not learned but inherent and therefore have an evolutionary origin.

To conclude, humans seem to have inherent prior beliefs. On one hand, there is the belief in the superiority of familiar alternatives. It makes us wrongly anticipate the expected utility of familiar and unfamiliar alternatives. On the other hand, we have prior beliefs about the ingroup and outgroup, affecting the attribution of their behaviour. Finally, although the outgroup homogeneity bias seems to be rather learned than inherent, its existence still appears to be in conflict with objective Bayesiansim. If we updated our beliefs correctly, group differences regarding perceived homogeneity should vanish at one point because obviously only one group can be more homogenous than the other (or they are equally homogenous). This is particularly true for groups we have a lot of interpersonal contact with such as the opposite sex. The fact that this does not happen (Park \& Rothbart, 1982) seems to imply that we do not update our beliefs according to Bayes' law. So, let us investigate this topic more closely in the next chapter.

\footnotetext{
${ }^{19}$ Unlike in case of the outgroup homogeneity bias, these different conclusions cannot be explained through more contact with ingroup than outgroup members and different categorisation when interacting with ingroup compared to outgroup members.
} 


\subsection{How We Update Beliefs}

The way we handle new evidence is essential in regard to the ultimate specification of our beliefs and therefore also the result of statistical discrimination. For example, if it were possible to hold certain beliefs despite substantial disconfirming evidence, almost any action could stem from statistical discrimination; You would only have to hold the respective beliefs. This would complicate the distinction between taste-based discrimination and statistical discrimination in empirical observations because what seems to be a taste might actually be a "strange" belief. Moreover, taste-based discriminators might hide their tastes behind some dubious beliefs. That is why we have to analyse how people update their beliefs more closely.

In a strict sense, the way we update beliefs can also be seen as a belief, namely the belief in how we should update beliefs. And if subjective expected utility theory assumes that humans are Bayesian updaters, it implies that updating beliefs employing Bayes' law is an inherent prior belief itself. In this chapter we examine whether humans exclusively update their beliefs by use of Bayes' law or whether there are other inherent prior beliefs about how we should update our beliefs as well. In so doing, we consider the remaining ten biases of table 4.1. We stick to the same approach as in the last chapter: (1) if possible bundle various biases that seem to be manifestations of the same inherent (updating) belief; (2) find an evolutionary ultimate explanation for the existence of the inherent belief; and (3) see whether these biases are universal so as to strengthen the argument that they truly are inherent and not learned. Again, it has to be highlighted that the last two goals are a rather speculative endeavour since in this area, research is often scarce.

In the first part, we look at how people deal with probabilities and how the concept of probability is connected to availability and frequency. Here, the following biases are relevant: availability heuristic, representativeness heuristic, illusion of truth effect, and illusory correlations. In the second part, we discuss the stereotypical bias and the hindsight bias. Both distort our memory and thereby might interfere with Bayesian updating. In the third part, we present the inherent prior belief that we are right and others wrong. Due to that we gather and process confirming evidence differently than disconfirming evidence and are less critical in regard to our own beliefs than those of others. This apparent circumstance of a systematic preference for our own beliefs is in conflict with Bayesian updating. The following biases are linked to it: confirmation bias, backfire effect, continued influence bias, and Semmelweis reflex. In the last part, we examine whether social 
identity affects our belief formation process, leading to beliefs that tend to flatter the ingroup and decry the outgroup.

\subsubsection{On Availability, Frequency, and Probability}

There are four biases in table 4.1 that all are somehow linked to probability: availability heuristic, representativeness heuristic, illusion of truth effect, and illusory correlations. The two basic assumptions behind these proximate mechanisms are simple. (1) Humans did not evolve to be good at handling probabilities but natural frequencies, "which simply report how many cases of the total sample there are in each subcategory" (Hoffrage et al., 2002, p. 346). (2) We use an incident's availability as a proxy for its natural frequency, whereby availability is mainly (but not exclusively) defined by the number of relevant instances and the ease with which these relevant instances come to mind (Kahneman, 2011).

How do these two assumptions interfere with Bayesian updating? Fischhoff and Beyth-Marom (1983) write: "To find a place in the Bayesian model, one's beliefs must be translated into subjective probabilities of the form appearing in the model. Any difficulties in assessing such component probabilities would impair hypothesis evaluation." (p. 244) At a later passage, the authors get more specific: "There is reason for concern whenever the assessors have followed procedures that are inconsistent with the rules of statistical inference. ... Two well-known deviations are reliance on the availability and representativeness heuristics when making probability assessments." (p. 245) Therefore, considering the two assumptions stated above, the law by use of which we actually update our beliefs looks as follows. First, we rewrite it so there no longer are probabilities but natural frequencies and for that use the formulation of Hoffrage et al. (2015). Note that $f\left(e \cap s_{i}\right)$ stands for the natural frequency of joint occurrences of $e$ and $s_{i}$, $f\left(e \cap \neg s_{i}\right)$ stands for the natural frequency of joint occurrences of $e$ and $\neg s_{i}$, and $f(e)$ for their sum.

$$
p\left(s_{i} \mid e\right)=\frac{f\left(e \cap s_{i}\right)}{f(e)}=\frac{f\left(e \cap s_{i}\right)}{f\left(e \cap s_{i}\right)+f\left(e \cap \neg s_{i}\right)}
$$

Second, we reformulate this theorem in order that availability serves as a proxy for natural frequency. Note that $a$ is an element of $\mathcal{A}$, which is the set of all functions that transform natural frequency to availability of natural frequency. Moreover, $a$ has to fulfil the condition that the posterior probabilities it produces 
satisfy the three assumptions of probability theory (cf. Kolmogorov, 1933).

$$
p\left(s_{i} \mid e\right)=\frac{a\left(f\left(e \cap s_{i}\right)\right)}{a(f(e))}=\frac{a\left(f\left(e \cap s_{i}\right)\right)}{a\left(f\left(e \cap s_{i}\right)\right)+a\left(f\left(e \cap \neg s_{i}\right)\right)}
$$

Now, let us begin with the first assumption: Humans did not evolve to be good at handling probability but natural frequency. Given this is true, it is not surprising that people perform badly at probability tasks, as for example the Linda problem. What is it about? Tversky and Kahneman (1983) conducted an experiment at various American universities, where they gave participants the following description: "Linda is 31 years old, single, outspoken and very bright. She majored in philosophy. As a student, she was deeply concerned with issues of discrimination and social justice, and also participated in anti-nuclear demonstrations." (p. 297) Now, subjects had to decide which of two statements is more likely: (a) Linda is a bank teller; (b) Linda is a bank teller and active in a feminist movement. Although (a) always has to be true if (b) is true but not vice versa, $85-90 \%$ of subjects chose option (b). Now, Gigerenzer (1997) argues that tasks intended to assess human statistical prediction should not present information in probability format but frequency format. ${ }^{20}$ The frequency format always involves frequencies as defined by a natural sampling tree (Gigerenzer \& Hoffrage, 1995). Multiple studies showed that this truly improves participants performance (Gigerenzer \& Hoffrage, 1995; Gigerenzer, 2002; Hoffrage \& Gigerenzer, 1998; Gigerenzer, et al., 1998; Lindsey et al., 2003; Gigerenzer et al., 2008). For example, in case of the Linda problem, the error rate decreased from $50-90 \%$ to $0-25 \%$ if the experimenters used a frequency format instead of a probability format (Fiedler, 1988; Hertwig \& Gigerenzer, 1999). ${ }^{21}$

What is the ultimate explanation for why we can handle natural frequencies better than probabilities? Haselton et al. (2015) say that "natural frequencies, such as the number of times an event has occurred in a given time period, are more readily observable in nature. In contrast, probabilities (in the sense of a number

\footnotetext{
${ }^{20}$ Here is an example of a task in probability format. The probability of breast cancer is $1 \%$ [base rate]; the probability of a positive test given breast cancer is $90 \%$ [sensitivity]; and the probability of a positive test given no breast cancer is $10 \%$ [false positive rate]. How many of those who test positive actually have breast cancer? Now, the same task in frequency format: Ten of every 1000 women have breast cancer; 9 of those 10 women with breast cancer will test positive and 99 of the 990 women without breast cancer will also test positive. How many of those who test positive actually have breast cancer? (Gigerenzer et al., 2008)

${ }^{21}$ Yet, some researchers such as Kahneman are not entirely convinced by these studies (see Mellers et al., 2001).
} 
between 0 and 1) are mathematical abstractions beyond sensory input data, and information about the base rates of occurrence is lost when probabilities are computed (Cosmides \& Tooby, 1996)." (p. 971) Moreover, to think of uncertainty as representations of mathematical probabilities was first devised in the $17^{\text {th }}$ century (Gigerenzer et al., 1989). Therefore, out of an evolutionary perspective, the concept of probability is brand new to us. No wonder that we are error prone when we solve respective problems.

We continue with the second assumption: In order to assess how frequent an incident is, we use its availability as a proxy. Of course, our ancestors could only depend on availability if it more or less resembled probabilities or if there was a good reason why it not necessarily had to. ${ }^{22}$ Otherwise, they would have constantly made suboptimal decisions due to misjudgements of probabilities. Whether availability and probability coincided is once again a question of the environment. For once, let us begin with today's environment. Kahneman (2011) presents the following impressive misjudgements: "Strokes cause almost twice as many deaths as all accidents combined, but $80 \%$ of respondents judged accidental death to be more likely. Tornadoes were seen as more frequent killers than asthma, although the latter cause 20 times more deaths. Death by lightning was judged less likely than death from botulism even though it is 52 times more frequent. Death by disease is 18 times as likely as accidental death, but the two were judged about equally likely. Death by accidents was judged to be more than 300 times more likely than death by diabetes, but the true ratio is $1: 4 . "$ (p. 138)

Why did participants perform so badly here? The answer is that the media reports way more often about deaths caused by accidents and tornados than deaths caused by asthma or diabetes (ebd.). This is because media coverage is not a simple representation of what is going on but biased towards novelty, oddity, extremity and poignancy. As a consequence, spectacular tornados and accidents are overly available and thus appear to happen more frequently than they actually are. Similarly, films such as "Jaws" let us shudder with fear and think twice whether we want to swim in the sea. Yet, shark attacks are very rare. Every year, there are only 70 to 100 shark attacks worldwide, of which 5 to 15 result in death. ${ }^{23}$ In a lifetime, the odds of getting attacked and killed by a shark are 1 in $3,748,067$. In fact, in the U.S., people are more likely to die from fireworks (1 in 340,733), lightning ( 1 in 79,746), drowning ( 1 in 1,134), a car accident ( 1 in

\footnotetext{
22 One such reason that is often stated involves that estimating probability via availability needs less cognitive resources than doing so via natural frequency (Tversky \& Kahneman, 1973; Kahneman, 2011).

${ }^{23}$ Just for comparison, humans kill around 100 million sharks a year (Zachos, 2018).
} 
84), stroke (1 in 24), or heart disease (1 in 5) (Florida Museum, 2018). Moreover, more people actually die from jellyfish stings than shark attacks (Muller, 2015). So, although shark attacks are highly improbable, they appear to be more frequent due to reports on shark attacks and especially films and books portraying shark attacks. Of course, the same mechanism applies if the media over and over portrays members of a social group in a certain way not because that portrayal is generally accurate but increases sales figures. ${ }^{24}$ Here, consumers of the media would again mistake availability for probability and thus overestimate the probability that the social group as a whole actually has the portrayed attributes. In summary, today, availability and probability do not always go together. Was that different in the late Pleistocene?

As mentioned in section 4.1.1, to determine the exact environment of our ancestors is very difficult. Thus, this paragraph is a hypothesis that needs further proof. Notwithstanding this limitation, if we go back 50'000 years, there certainly was no worldwide media which could distort a possible relationship between availability and probability. Admittedly, there probably were myths and stories that the elderly told the youngsters which might have led to wrong probabilities too. Yet, in all likelihood, these myths / stories were rather local and therefore also relevant for the group. Thus, if you were frequently told something, you should not ignore it because it probably affects your life. In contrast, today, much news is irrelevant for our personal life. Of course, a terroristic attack is immensely tragic. However, the chances to be affected by one is marginal, especially if you live in a country that has not had such an attack yet. Still, due to the high international media attention that terroristic attacks provoke, they become highly available which is why we ultimately bother about them. This would not have been the case in the late Pleistocene because back then, we had no chance to hear from things that happened hundreds of kilometres away in the first place.

If we accept the above argument, we agree that while 50'000 years ago high availability might not necessarily have involved high probability, it should have involved high relevance. Today, due to international media, this no longer has to be true. But how can we explain the gap between availability and probability that might have already existed in the late Pleistocene? Sunstein (2005) argues that people use availability in order to assess the magnitude of risk which a certain action involves. His idea is as follows: "If a particular incident is cognitively "available"- both vivid and salient - then people will have a heightened fear of

\footnotetext{
${ }^{24}$ It is already sufficient if one medium does that given the consumers of this medium do not consume another medium. Thus, it is not necessary that the media in general portrays an inaccurate impression of a certain group.
} 
the risk in question." (p. 77). In turn, this fear leads us to neglect the actual probability of an incident (Sunstein \& Zeckhauser, 2011). Consequently, he implies that high availability is connected with fear of a risk and thereby high potential costs. But why should it be adaptive to overestimate the probability of a costly incident through making it overly available and fearing it?

This is where error management theory (Haselton \& Nettle, 2006) comes into play again. Some situations might be rare but very costly if they occur, leading to an asymmetry between false negative and false positive. In order to illustrate that let us assume that a hunter-gatherer group goes fishing at the same spot for several years. One day, a member is attacked by a shark and dies. The group can draw different inferences out of this event. (1) Since this happened the first time in several years, it is improbable that it will happen soon again. Thus, we continue fishing there. (2) We do not know whether this incidence was simply bad luck or long overdue. So as to find that out we need more data and thus continue fishing there. (3) We do not know whether this incidence was simply bad luck or long overdue. Since it is too risky to find it out we stop fishing there. (4) If it happened once, it is probable that it will happen again. Thus, we stop fishing there. First of all, let us assume that leaving the fishing spot does not automatically imply that the group will starve to death. So, food is relatively abundant. Now, if the group leaves the spot and starts fishing somewhere else although there would not have been another shark attack, the costs of that error are relatively small. However, if the group does not leave the spot because they think that it is safe or want to gather more data and another shark attack occurs, this error is very costly. As a consequence, if a certain outcome is relatively unlikely but very costly, following availability and not probability can be fitness enhancing, even if the two might diverge.

Now, the tendency that we mistake availability for probability is of course at the very heart of the availability heuristic. As previously mentioned, Sunstein (2005) says that the purpose of the availability heuristic is to assess the magnitude of risk that a certain action involves (e.g. fishing at spot X). Thus, it is not about how probable a risk is but how available it is in one's mind and social environment. In contrast, the representativeness heuristic says that when we assign people to categories, we mainly consider how well they match the prototype of the category and neglect the base rate (Kahneman, 2011). So, again, we do not think in probabilities but availability. If trait $\mathrm{X}$ is highly available in case of group A but unavailable in case of group $\mathrm{B}$, we assume that someone that has trait $\mathrm{X}$ should be part of group A. In so doing, we neglect the possible circumstance that group $\mathrm{B}$ is much larger than group $\mathrm{A}$ and thus altogether might actually have more members with trait $\mathrm{X}$. 
The deliberations of this chapter also provide an explanation for the illusion of truth effect. We said that if in a hunter-gatherer society you are told something multiple times, it is probably relevant. In this way, the repetition of a statement makes it more available, which is why we also think that it is more probable. Finally, illusory correlations are the result of availability combined with or produced by cost-asymmetric false positive and false negative errors. What does that mean? First, illusionary correlations can be fitness enhancing if the costs of incorrect assumptions are rather small, whereas the benefits of the occasional correct assumption are rather large (Foster \& Kokko, 2009). This applies to all situations where the current data suggests a correlation, however, you have too few data to make a proper prediction. For example, someone catches a fish from a newly found lake and eats it. The next day, she is dead. Now, in order to say with (almost) certainty that the fish caused her death and that other fish of that lake would do so as well, more people would have to catch and eat fish from that lake. Yet, given the fish truly killed her and the other fish would have done so as well, this elimination of alternative explanations would come at a high price. Therefore, it is more fitness enhancing to directly assume that there is causality, even if chances are actually high that there is none. ${ }^{25}$ The result of this is that we follow availability instead of probability because probability based on a sample of one incident is more or less meaningless. This also explains why distinctiveness (minority group) and particularly double distinctiveness (minority group and negative behaviour) lead to illusory correlations (Hamilton \& Gifford, 1976). Distinctiveness is equivalent to salience and high salience provokes high availability. In turn, if something is highly available we might overestimate its probability. In case of double distinctiveness, this also makes sense out of the following reason: Our sample of minority group members' behaviour is smaller than that of majority group members. So, every new information about a minority group member's behaviour is generally more valuable than that about a majority group member's behaviour. ${ }^{26}$ This is why new information about the minority group is generally more salient than new information about the majority group. This first distinctiveness is then combined with the second one: The costs of not finding the correlation between cause and effect are higher if the effect is

\footnotetext{
25 Today, we still see this tendency if we look at lucky charms. For example, if you write your first exam at the university with a certain pen and the exam went really great, you might want to write the next exams with this pen as well because you think that it brought you luck. You do not start a little experiment where you write half of the exams with your lucky pen and the other half with a different pen so as to examine whether your lucky pen truly boosts your performance.

${ }^{26}$ Normally, the larger the sample, the better it resembles the true mean and variance.
} 
negative compared to positive, provided that our survival is not already seriously endangered (cf. negativity bias). Both together create an illusory correlation.

To finish this chapter, let us examine whether these biases are universal. First of all, Sunstein (2005) says that the availability heuristic can be detected in different cultures and it partly still serves its original function of emphasising risks. He writes: "The availability heuristic helps to account for ... cross-national differences [in risk perception of specific incidents] and for generally exaggerated risk perceptions." (p. 91) Yet, it has to be mentioned that his analysis only includes American and European culture, which of course limits the universal claim of the availability heuristic. Unfortunately, there is no study that examines the existence of the availability bias in other cultures such as East Asian or South American cultures. Yet, there is one hint which reveals that people from these cultures also have an availability heuristic. After the terrorist attacks in Europe in 2015/2016, many tourists cancelled their trips to France even if their travel destination was far away from these attacks. Here, tourists from Western cultures were not more prone to do so than tourists from non-Western cultures (Alderman, 2016). Actually, in case of the Louvre, especially non-Western tourists stayed away. While the number of American visitors remained stable in 2016, the museum welcomed $61 \%$ fewer Japanese, $53 \%$ fewer Russians, $47 \%$ fewer Brazilians and $31 \%$ fewer Chinese (Willsher, 2017). These numbers suggest that availability of a certain risk is more influential on decision-making than the actual probability of that risk.

There is one cross-cultural study regarding the representativeness heuristic, conducted by Spina et al. (2010). The study involved Canadian and Chinese participants. Yet, the authors did not examine the role of representativeness in a social categorisation context as we did in this chapter but in the context of assigning cause and effect. This so-called cause-effect magnitude correspondence implies that big causes (e.g. shark attack) are more likely to lead to big consequences (e.g. death) than small causes (e.g. mosquito sting). ${ }^{27}$ While Spina et al. found that there was an overall tendency to follow the cause-effect magnitude correspondence, this tendency was significantly stronger in case of Canadian subjects. The authors' explanation for this difference involves the cultures different degree of holistic thinking. Accordingly, if Canadians were primed to think more holistically, they displayed less cause-effect correspondence. Thus, culture affects this aspect of the representativeness heuristic. Yet, since participants of both cultures 27 The same is true in the opposite direction. So, big effects should be the product of big
causes. 
revealed a cause-effect correspondence, this overall tendency seems to be culture invariant. Finally, it is unclear whether these findings can be applied on the representativeness heuristic in general.

Although there is no cross-cultural study about illusory correlations it is widely known that superstitious beliefs are not limited to Western cultures. Unlucky numbers provide a perfect example. For instance, the German airway company Lufthansa does not have a seat row with the number 13 (unlucky number in many Western countries) or 17 (unlucky number in Italy and Brazil). In contrast, the Japanese airway company All Nippon Airways does not have a seat row with the number 4,9 , and 13 . This is because 4 and 9 are unlucky numbers in Japan (Tingler, 2010). There is even a word that describes the fear of the number 4 : tetraphobia. It is most common in East Asian countries. That is because in these languages the pronunciation of the number 4 is similar to that of the word death (Havil, 2010). Thus, while the exact manifestation of an illusory correlation is highly affected by culture, the phenomenon per se seems to be universal (Foster \& Kokko, 2009; Laland \& Brown 2002; Richerson \& Boyd 2005).

Again, there is no cross-cultural study about the illusion of truth effect. Unfortunately, there also seems to be no study that uses non-Western subjects. Indeed, there is one Chinese study ( $\mathrm{Li}$ et al., 2016) about rumour spreading and the illusion of truth effect. However, this study is exclusively computational and does not have any participants. Yet, despite the lack of empirical data, the fact that the authors acknowledge the illusion of truth effect might be seen an indication for its existence in China. ${ }^{28}$ Or, at least, the illusion of truth effect does not appear counterintuitive to them. But notwithstanding these deliberations, since there is no cross-cultural data we have to infer that the influence of culture on the illusion of truth effect is unknown.

To summarise, we discussed a twofold ultimate explanation in this chapter. First, we are bad at probability tasks because in the course of evolution we were almost exclusively confronted with natural frequencies and not probabilities. Second, we use an incident's availability as a proxy for its natural frequency. Because of the locality of information in the late Pleistocene, availability, relevance, and probability seem to have coincided more than today. Yet, gaps between availability and probability might have still existed. Error management theory is

\footnotetext{
${ }^{28}$ Admittedly, this is a rather weak argument because it could also be argued that the very fact that there are no empirical studies about the illusion of truth effect in Asian countries indicates its irrelevance there.
} 
able to provide an explanation for these. Lastly, while cross-cultural studies are rare, it does not seem that the four biases discussed in this chapter have a cultural origin.

\subsubsection{Distorted Memories}

In section 4.1.1, we have already discussed two biases that are linked to memory: rosy retrospection and the choice supportive bias. There, we said that they contribute to the inherent belief in the superiority of familiar alternatives. Of course, these two biases might also influence the way we update our beliefs. Since we whitewash possible decision errors through reattributing the past and past choices in an overly positive way, there are no apparent mistakes we can learn from. In turn, this prevents us from adjusting our beliefs. The two biases in this chapter, namely the stereotypical bias and the hindsight bias, seem to affect Bayesian updating as well, yet, one might also be the very product of it.

Let us start with the stereotypical bias, which implies that stereotypes distort our memory (Payne et al., 2004). Note that a stereotype is ,a cognitive structure that contains our knowledge, beliefs and expectancies about some human social group" (Pendry, 2015, p. 96). Therefore, we use stereotypes synonymously with group specific beliefs. Now, the stereotypical bias appears to have the following source of error: In hindsight, we apply a stereotype on an individual although we should know from experience that this individual did not behave in a stereotypical way (Payne et al., 2004). More technically spoken, a situation actually reveals stereotype inconsistent evidence $e$, yet, the stereotypical bias modifies $e$ in such a way that we perceive / remember evidence $e^{\#}$, which is stereotype consistent.

Let's illustrate this by use of a classic study conducted by Allport (1947). He showed subjects a scene depicting a black man and a white man arguing on a tram. The white man held a razor in his hand. After several retellings from one subject to another, Allport reports that "[i]n over half of the experiments with this picture, at some stage in the series of reports the Negro (instead of the white man) is said to hold the razor in his hand" (p. 111). So, at some point in the retelling chain, the actual evidence $e$, which involves that the white man holds the razor, turned into the stereotype-consistent evidence $e^{\#}$, where the black man holds the razor.

We can think of three reasons why this happened. First, a subject has a total lapse of memory regarding who holds the razor and thus simply fills it with a stereotype, which says that the black person holds it. Second, a subject truly believes that the black person holds the razor because she reminds it that way. 
Third, a subject actually knows that the white person holds the razor, yet, for some reason says that the black person holds it. Since this last possibility involves conscious misdirection we only concentrate on the first and second reason in the further course.

Let us first investigate why we fill a lapse of memory with a stereotype. If you have a lapse of memory regarding a certain evidence $e$, you can handle it in three ways: a) acknowledge that you have a lapse of memory and therefore no clue about the evidence $e$; b) fill the lapse of memory with stereotype consistent content; and c) fill the lapse of memory with stereotype inconsistent content. Now, option c) seems very illogical. Filling the lapse of memory with stereotype inconsistent rather than consistent content means that you use "filling material" that you subjectively do not perceive as most suitable and thus most probable. For example, let us assume you once asked a medicine student what she wants to do after her studies but now you cannot recall what she said. Of course, most probably she becomes a doctor. Therefore, on general, it is much more accurate to fill this lapse of memory with "she wants to become a doctor" than with "she wants to do a second degree in law". ${ }^{29}$

The question that remains is why we do not simply acknowledge the lapses of memory and leave it unfilled. First of all, sometimes if not frequently we do acknowledge that our memory is imperfect and have no clue about a certain evidence $e$. So, this is not like our blind spot in the eye that we constantly fill with apparently suitable information. We do not have the illusion of a perfect memory as we have an illusion of a perfect field of vision. Second, filling a lapse of memory with stereotype consistent content can be fitness enhancing. This is the case if the costs of not having any information are higher than the costs of potentially assigning the wrong content to the lapse of memory. These potential costs of incorrect stereotype assignment highly depend on how accurate the stereotype generally is. This means the more accurate the stereotype, the better the stereotype consistent content should suit the lapse of memory. In turn, the better the fit, the lower are the chances of error and thus the overall costs. Actually, these deliberations are very similar to the ones on why we make use of statistical discrimination: It helps us to better handle uncertainty. Now, in case of a lapse of memory, we could also say that there is complete uncertainty about whether evidence $e$ was present or not. Due to that one might therefore exclude evidence

${ }^{29}$ Of course, this does no longer have to be the case if you know that she is actually very unhappy with her studies and highly interested in law. Yet, if you knew that you probably would not compare her with the prototypical medicine student in the first place. 
$e$ when making predictions. Yet, we not always behave in this way because excluding evidence $e$ when making predictions might make us less able to react on our environment. Using the words of Macrae and Bodenhaus (2000), we want our environment to be a predictable place. This does not only apply for our present and future but also past environment. Accordingly, we sometimes fill our lapses of memory in the most predictable way, which means with stereotype consistent content.

Let's continue with the second reason: We truly believe that a certain stereotype inconsistent evidence $e$ was actually stereotype consistent and thus remember it (and maybe have also perceived it) as $e^{\#}$. The explanation behind such wrong recollections (and perceptions) might lie in the process of categorisation more generally. This is because categorisation might not only be essential for social perception but perception in general. Barrett (2017) describes the process of categorisation as follows: We compare the sensory input with our concepts ${ }^{30}$, apply the concept that fits it best, form predications, and, in this way, make the sensory input meaningful. This process can lead to mistakes, called prediction errors. There are two ways to solve them. The first one is to change our predictions and adjust them to the sensory input until they match. The second one is to keep the original predication and filter "the sensory input so it's consistent with the prediction" (p. 64).

This second handling of prediction errors could explain what happened in case of subjects who misremembered (and also misperceived) which person held the razor. By categorising the retelling of the depicted scene, they predicted that the black and not the white man holds the razor. This prediction error did not get corrected via adjusting the prediction but via adjusting the sensory input (the memory). As a result, they thought that they had truly heard that the black person holds the razor and therefore also remembered it like this.

We see that the stereotypical bias can be described as a by-product of categorisation. In turn, categorisation in the sense of Barrett (2017) can be described as predictive coding or predictive processing, which is a Bayesian approach to brain function (Clark, 2013, 2015; Friston, 2010, 2012). As a consequence, the stereotypical bias does not have to be in conflict with Bayesianism. However, the circumstance that our beliefs and their consequent predictions can filter the sensory input implies that two people facing the same evidence $e$ can perceive /

${ }^{30}$ A concept involves the knowledge / beliefs we have about a category. 
remember it differently if they have different priors. ${ }^{31}$ And their diverging perception of $e$ might never converge given the prediction error is always handled via adjusting the sensory input. ${ }^{32}$ Nevertheless, some sensory input that interferes with our predictions is very unlikely to be (constantly) filtered out. As Clark (2015) writes: "[W]e are not slaves to our expectations. Successful perception requires the brain to use stored knowledge and expectations (Bayesian priors) to minimize prediction error. But we remain able to see very (agent-) surprising things, in conditions where the brain assigns high reliability to sensory prediction error (hence high reliability to the driving sensory signal)." (p. 79) Admittedly, the assessment of a sensory prediction error's reliability can be distorted as for example in case of mental illnesses or drug use (ebd.). Moreover, it seems to be improbable that evolution led to a perceptual apparatus that is as accurate and therefore veridical as possible (Hoffman et al., 2015), indicating that we might constantly assign wrong reliabilities to some sensory prediction errors (cf. error management theory (Haselton \& Nettle, 2006)). Yet, in these cases this concerns all people since humans should have "learned" to assign these wrong reliabilities in the course of evolution, making it an inherent prior belief. Therefore, someone who holds a certain group specific belief despite substantial disconfirming evidence (that others are able to perceive) might theoretically do so because he constantly filters incoming sensory information in such a way that it still matches his predictions. Nonetheless, it appears a lot more likely that he is able to maintain this belief due to a non-Bayesian updating process and / or a group specific inherent prior belief.

After having discussed the stereotypical bias, let us continue with the second bias of this chapter, namely the hindsight bias. The hindsight bias involves the phenomenon that after we know the outcome of an event we tend to overestimate the predictability of this outcome in foresight (Hoffrage \& Pohl, 2003). In technical terms, the hindsight bias appears to have the following implications: After considering evidence $e$, the prior $p\left(s_{i}\right)$ that gets updated to the posterior

\footnotetext{
${ }^{31}$ Subjective Bayesians can have different priors due to differently assigned first priors where they had not faced any (relevant) evidence yet or due to different evidence they faced in the past. Objective Bayesians can have different priors due to different evidence they faced in the past. In contrast, objective Bayesians that faced the same evidence in the past must come to the same posterior subjective probability after facing new evidence $e$ (Strevens, 2006).

32 A picture called "The dress" provides an example of this. It displays a dress that some people perceive as gold and white and others as black and blue. The dress actually is black and blue but this knowledge does not change the perception of those who perceive it as gold and white in the picture (MacFarquhar, 2018). For Jay Neitz, who has been studying individual differences in colour vision for 30 years, "The dress" provokes one of the biggest individual differences he has ever seen (Rogers, 2015).
} 
$p\left(s_{i} \mid e\right)$ is remembered as having been closer to $p\left(s_{i} \mid e\right)$ than it actually used to be before considering evidence $e$. Such a tendency interferes with Bayesian updating (Madaráz, 2011; Mahdavi \& Rahimian, 2016). Moreover, it can be harmful. As Fischhoff (1982) writes: "The very outcome knowledge which gives us the feeling that we understand what the past was all about may prevent us from learning anything from it" (p. 343) Thus, if we always say "I knew it all along" even though we did not, we might not update our beliefs appropriately, which can lead to inaccurate predictions and thereby suboptimal decisions.

So, if a hindsight bias appears to exacerbate adaptive learning, why do we still find it all over the world (Pohl et al., 2002)? Hoffrage et al. (2000) argue that the bias is actually a very by-product of knowledge updating. When we get informed about the outcome of an event, we might simultaneously update the knowledge we used so as to form our prediction. Given this occurs without much conscious notice, we now base our hindsight prediction on updated knowledge which is why we think that we knew it all along. Additionally, if we cannot retrieve our original judgment, we have to reconstruct it. By doing so, we again go through the same steps of inference which produced the original judgement. Yet, some cues that were missing in case of the original judgement are now known (Todd et al., 2005). As a result, this new judgment is closer to the actual outcome than the original judgement. Hoffrage et al. (2000) confirm this hypothesis. They found that feedback on an outcome of an event cannot only affect recalled prediction but also the memory of variables that are associated with that event. To summarise, "[o]nce an additional event occurs, our knowledge is updated to reflect this new information and our knowledge after feedback becomes systematically shifted towards the new, updated reality. Thus, when the decision maker has to recall an earlier judgment in the future, the recalled judgment will be closer to the outcome of the new event than to the original judgment." (Haselton et al., 2009, p. 740)

The last paragraph provided proximate explanations for the hindsight bias. The ultimate question of why this is adaptive is still unanswered. There are two non-exclusive ultimate explanations in the literature. First, continuously adjusting past information to more recent one efficiently avoids capacity problems (Bjork \& Bjork, 1988; Schacter, 1996). Second, this adjustment may also improve our inferences over time (Hoch \& Loewenstein, 1989; Hoffrage et al., 2000). This is because the hindsight bias leads to constant up-to-date knowledge in an ever-changing environment (Todd et al., 2005). ${ }^{33}$ The circumstance that the bias decreases the more experience people have with the task under consideration is

\footnotetext{
33 This statement inheres the assumption that our ancestors' environment was unstable enough in order that constant up-to-date knowledge which to some degree ignored previous knowledge became adaptive. As mentioned in section 4.1.1, it is rather pointless to discuss whether our
} 
said to confirm the "better-inference-hypothesis" (Hertwig et al., 2003; Todd et al., 2005; Christensen-Szalanski \& Willham, 1991). The idea is that, normally, the more comprehensive our knowledge in foresight, the less influential becomes an additional piece of information. Consequently, after the outcome of an event is known, experienced people do not have to update their knowledge as strongly as inexperienced people.

Hedden (2019) takes a different explanatory approach of the hindsight bias, namely that it is not (always) a bias in the first place. While he acknowledges that the hindsight bias is not compatible with Bayesianism and thus not rational in a Bayesian sense, he questions the very concept of ideal rationality defined by Bayesianism. More precisely, he argues that the necessity of logical omniscience, which Bayesianism assumes for ideal rationality, is mistaken. ${ }^{34}$ Given we abandon it, the hindsight bias can become rational. This is because "[t]he truth of a hypothesis often provides evidence about what the evidence available ex ante was, and also about what that ex ante evidence supports. So often, upon learning that the hypothesis is true, you should become more confident that the ex ante evidence strongly supports that hypothesis and also increase your expectation of the degree to which it does so." (p. 50)

All in all, we see that the hindsight bias appears to be a by-product of nonBayesian knowledge updating. It can either be explained via an evolutionary approach that depicts the bias as fitness enhancing or via rejecting the Bayesian assumptions of ideal rationality which in turn enables the bias to become rational.

To summarise this chapter, both the stereotypical bias and the hindsight bias seem to interfere with Bayes' law. On one hand, we fill our lapses of memory with stereotype consistent content and filter our perception / memory so that it becomes stereotype consistent. On the other hand, after we know the outcome of an event we tend to overestimate the predictability of this outcome in foresight. Yet, while the hindsight bias appears to truly be incompatible with Bayesian updating, the stereotypical bias can actually be explained via a Bayesian approach and therefore does not have to interfere with Bayes' law.

ancestors' environment truly provided such conditions. The only thing we know for sure is that their environment was not completely stable (e.g. Martrat et al., 2004).

34 This is a common critique of Bayesian epistemology (cf. Talbott, 2008). 


\subsubsection{Why We Defend Our Beliefs}

The remaining four biases of table 4.1 make us gather and process belief confirming and belief disconfirming evidence in a non-neutral way. As a consequence, they prevent us from adjusting an apparently wrong belief even if there seems to be ample evidence that disconfirms it. We can formalise this as follows. Note that $p\left(s_{+}\right)$is the belief or more precisely the subjective probability we want to defend. Moreover, $\alpha>1$ and $0<\delta<1$, whereby $\alpha$ and $\delta$ stand in such relation to each other so that the posterior probabilities they result in fulfil the three assumptions of probability theory (cf. Kolmogorov, 1933).

$$
p\left(s_{+} \mid e\right)=\frac{\alpha p\left(s_{+}\right) p\left(e \mid s_{+}\right)}{\alpha p\left(s_{+}\right) p\left(e \mid s_{+}\right)+\delta p\left(\neg s_{+}\right) p\left(e \mid \neg s_{+}\right)}
$$

In this way, after the decision-maker has considered new evidence $e$, the posterior probability of $s_{+}$is higher as it should be. The respective biases that lead to this outcome are: confirmation bias, backfire effect, continued influence bias, and Semmelweis reflex.

At first sight, these cognitive distortions might be somewhat surprising out of an evolutionary perspective. Indeed, we have to reconstruct our environment on a simpler model before we can manage it (Kite \& Whitley, 2016). However, on a given level of simplification, why does this reconstruction have systematic distortions? We want the environment to become a predictable place which we can react on and interact with. Accordingly, if new evidence seems to disprove our beliefs, aren't we better off by taking this new evidence seriously and including it into our model of the environment? Wouldn't that lead to better predictions and ultimately more fitness?

A proximate explanation of why we defend our beliefs that is often mentioned involves the tendency that humans are verifiers and not falsifiers (Mercier \& Sperber, 2017). So, unlike critical rationalism of Popper (1963) proposes, our approach to check a hypothesis is not to falsify it but to try to verify it. Wason (1960) provided first evidence for this and thereby built the corner stone of the confirmation bias. He inferred: "[T]here would appear to be compelling evidence to indicate that even intelligent individuals adhere to their own hypotheses with remarkable tenacity when they can produce confirming evidence for them." (Wason, 1977, p. 313) Now, the decisive word in the last sentence is "own". So, it is true that as soon as we have chosen a position regarding an issue we are good at producing arguments that confirm / verify this position but rather bad at producing 
counterarguments (e.g. Kuhn, 1991). This is why $\alpha$ is larger than 1 in the above formula. However, if we then are confronted with an opposite position, we are in turn good at producing counterarguments that falsify it and bad at producing arguments that verify it (Shaw, 1996; Mercier \& Sperber, 2011). This is why $\delta$ is smaller than 1 . Therefore, we do not generally have a preference for testing hypotheses via verification / confirmation. This is only true for the positions / beliefs we hold. Given someone challenges us with an opposite position, we preferably look for counterarguments that falsify this opposite position. This is why Mercier and Sperber (2017) speak of a myside bias rather than a confirmation bias.

Of course, this clarification has not solved the problem of an ultimate explanation. Nevertheless, the myside bias quite suitably encompasses the four biases mentioned above in superordinate manner. Despite the presence of disconfirming evidence, we hardly adjust a wrong belief because: (1) We are good at producing arguments that still confirm it; and (2) we are good at producing counterarguments for the disconfirming evidence and hereby mitigating the power of the disconfirming evidence. Thus, if we find an ultimate explanation for the myside bias, we indirectly also find an ultimate explanation for the four biases mentioned above. ${ }^{35}$

How could this ultimate explanation look like? If we examine the myside bias from an individualistic perspective, it is hard to find an evolutionary argument for its existence. Why should defending prior beliefs that face substantial disconfirming evidence be fitness enhancing? Let us consider the following example: I believe that river $\mathrm{X}$ is the best river for fishing because it is the richest in fish. So, $I$ fish at river $X$ and catch between one and three fish a day. Then, a family member tells me that river $\mathrm{Y}$, which is equally far away as river $\mathrm{X}$, is much richer in fish than river $X$ and that she catches between three and five fish a day there. Now, I can either explain the difference in caught fish by reasoning that river $Y$ has more fish than river $\mathrm{X}$ and thus adjust my belief that river $\mathrm{X}$ is the richest in fish. Or I can stick to my belief that river $\mathrm{X}$ is the richest in fish and look for other explanations, such as the person is lying, was only lucky, or I simply had bad luck the last few days and would normally catch between five and seven fish. In this situation, stubbornly sticking to my belief and not even checking out river $\mathrm{Y}$ seems not to be fitness enhancing. Accordingly, studies about animal behaviour could also not find a confirmation bias (Mercier \& Sperber, 2017). For

\footnotetext{
35 The ultimate explanation for the myside bias presented in this subchapter will be mainly based on Hugo Mericer's and Dan Sperber's interactionist approach and their book "The Enigma of Reason" (2017).
} 
example, animals abandon their food patches the moment they expect to find better elsewhere (Pyke, 1984). ${ }^{36}$ So, why do humans but not animals have a bias here?

A major difference between humans and animals is our highly developed ability to communicate with each other. This ability or more specifically its consequences might be the reason why we suffer a myside bias, whereas animals do not: We should not examine reasoning from an individualistic perspective but from an interactionist perspective. As a consequence of this change in perspective, the primary goal of reasoning is no longer to individually (as a lone reasoner) find the most accurate belief but to convince others from your belief. In this case, a myside bias makes perfectly sense because it primarily leads to arguments that confirm your position and disconfirm opposite positions. This is particularly advantageous in two contexts. In a competitive context, a comparison of one-sided arguments helps to extract which of the options that competitive parties propose is best. For example, there are two parties within a group. One wants to settle down at river $\mathrm{X}$, the other at river $\mathrm{Y}$. In the end, the more convincing arguments will prevail and the consequent options be chosen. However, it has to be emphasised that argumentation is not exclusively a zero-sum game, leading to a winner and a loser. In a constructive context, the comparison of one-sided arguments serves as an advantageous method for finding the best position. For instance, a group faces an ambiguous situation and forms two parties. One has to argue for option $\mathrm{A}$, the other for option B. This saves resources because an individual does not have to assess both options. Therefore, Mercier and Sperber (2017) infer: "The myside bias doesn't turn argumentation into a purely competitive endeavor. Argumentation is a form of communication and is typically pursued cooperatively. At its best, the myside bias becomes a way of dividing cognitive labor." (p. 221)

The sole fact that parties compete against each other with their one-sided arguments does not automatically lead to accurate beliefs (which would make the world more predictable). This is only true if the most convincing argument is also the most accurate argument. Thus, while the myside bias indicated what kind of arguments we produce, we now have to examine how we evaluate arguments.

At first sight, our evaluative qualities do not seem very promising. Several studies such as Kuhn (1991), Nisbett and Ross (1980), and Perkins (1985) found that when experimenters asked participants why they hold a certain belief, their reasons were quite superficial and weak. So, people's criteria for their own reasons seem to be rather lax. This would pose a problem if we followed an individualistic

\footnotetext{
${ }^{36}$ Admittedly, it is unclear what "expect" precisely means in case of animals. Yet, Mercier and Sperber use this word.
} 
perspective. However, in real life, argumentation typically occurs in a dialogic context. Thus, when we use a weak argument our counterpart does not simply write it down and asks whether we have further arguments (as the experimenter does) but challenges it. Through this interactive back-and-forth, weak arguments should vanish and strong arguments prevail, provided the following two requirements are fulfilled: (1) Our counterpart's criteria for our reasons have to be rather rigid, otherwise we would not be challenged. (2) We have to acknowledge the superiority of certain arguments even though they are not ours.

Let us start with the first requirement. Trouche et al. (2016) conducted a sophisticated experiment that wanted to reveal the asymmetry between how critically we evaluate our arguments and those of others. The experiment had three phases. In the first phase, participants had to solve five reasoning problems concerning the products sold in a fruit and vegetable shop. For example, they were told that a fruit and vegetable shop carries, among other products, apples of which none are organic. Then, subjects had to infer as quickly and intuitively as possible which of the following statements applies for sure: "All the fruits are organic"; "None of the fruits are organic"; "Some fruits are organic"; "Some fruits are not organic"; and "We cannot tell anything for sure about whether fruits are organic in this shop". There was always one correct answer (here it would be "Some fruits are not organic"). In the second phase, participants had to explain why they solved each problem the way they did. By doing so, they got the chance to change their answer(s) if they realised that their reasoning was flawed. In the third phase, subjects were again given the five problems, one by one, with a reminder of their answer of the first phase. Additionally, they were told that another subject, who completed the experiment earlier that day, answered the problems differently and participants were also displayed the explanation of that differently thinking prior subject. Again, they got the chance to adjust their original answer if they wanted to.

Now, the third phase had two conditions. In one condition, the experimenters truly gave participants their own answers and an answer that differed from their own. However, in the other condition, the experimenters manipulated the reminder of the participant's answer given in phase one. So, in this condition, participants were not shown their actual answer. In turn, the prior subject, who was said to have answered the problems differently than the participant, now answered the problems in the way participants did in the first phase and thereby also used their explanations. By means of this design, the authors could examine whether subjects are more critical with their own reasoning when they think it actually is someone else's compared truly their own. 
The results are as follows: In phase one, participants answered 2.9 of the five problems correctly. Consistent with the myside bias, in phase two only few (approximately $14 \%$ ) changed their minds. These changes were as likely for the better as for the worse. In the third phase, $46 \%$ of those whose answers were manipulated noticed the manipulation, whereby those who gave the correct answer in the first phase had a significant higher detection rate. In case of those who could be successfully misled, $42 \%$ adjusted their misled answer to their prior one. In other words, $58 \%$ declined their own answer, which they defended in the second phase of the experiment. While the acceptance of the misled answer was positive for $2 / 3$ of participants (they switched from an invalid answer to a valid one), for $1 / 3$ it was negative (they switched from a valid answer to an invalid one). So, the contrast between the second and the third phase reveals that participants evaluated the quality of their own argument more rigidly when they thought it is someone else's (third phase) vs. their own (second phase). Accordingly, Trouche et al. (2016) infer that "people are more critical of other people's arguments than of their own". (p. 2122)

Mercier and Sperber (2017) call this tendency selective laziness. It means that people are lazy when they control the quality of their own argument but demanding when they do so in case of someone else's argument. Let's again look at the formulation at the beginning of this chapter.

$$
p\left(s_{+} \mid e\right)=\frac{\alpha p\left(s_{+}\right) p\left(e \mid s_{+}\right)}{\alpha p\left(s_{+}\right) p\left(e \mid s_{+}\right)+\delta p\left(\neg s_{+}\right) p\left(e \mid \neg s_{+}\right)}
$$

We can integrate selective laziness into it employing the same variables we used in case of the myside bias. Our laziness in controlling the quality of our own arguments can be represented by $\alpha>1$. In turn, our rigidity regarding arguments of others can be represented by $0<\delta<1$. The authors argue that selective laziness is adaptive because the process of finding strong arguments requires plenty cognitive resources. Therefore, we begin with a rather resource-poor but probably also weak argument and await whether our counterpart already accepts it. If she does, we do not have to invest further cognitive resources so as to find a better argument. If she does not, we have to find a better argument and if we do not find one, accept hers.

Let us continue with the second requirement: We accept the superiority of certain arguments. The experiment of Trouche et al. (2016) discussed above already suggests that this is true. If the superiority of the argument were meaningless, participants should have equally often declined the misled answer and changed to 
their actual own one, regardless whether their own argument was valid or invalid. Yet, this was not the case. $57 \%$ adjusted their misled answer and changed to their prior argument of phase one if that argument was valid. Meanwhile, only $31 \%$ did so if their prior argument of phase one was invalid. So, a valid (counter)argument made more participants change their answer than an invalid one, demonstrating the acceptance of a superior argument.

In a series of experiments, Trouche et al. (2014) further examined this topic. For example, participants had to solve the following problem: "Paul is looking at Linda and Linda is looking at John. Paul is married but John is not. Is a person who is married looking at a person who is not married?" The possible answers were "Yes"; "No"; and "Cannot be determined". The modal answer typically is "Cannot be determined" (cf. Toplak \& Stanovich, 2002). This answer is of course wrong. Consider the following argument: Linda is either married or not married. If she is not married, then Paul, who is married, is looking at her, so the answer is "Yes". If she is married, then she is looking at John, who is not married, so the answer is again "Yes". Therefore, no matter whether Linda is married or not the answer is always "Yes." After participants committed themselves to a (mostly wrong) answer, they were presented this argument. More than half immediately changed their minds. ${ }^{37}$ In order to exclude the possibility that participants simply adopted the provided argument because it came from the experimenters, the authors told them that the argument was given by a prior subject. To one group, the experimenters even said that this prior subject was really bad at such tasks. Still, most accepted it. This was also true if participants were told that this prior subject would earn some money if others get the problem wrong. So, despite not trusting the prior subject, they acknowledged the superiority of her argument. Another group had to think hard about the problem and justify their answer. Although only few got it right, most of them indicated that they were extremely confident about their answer. Yet, this confidence did not make them change their answer less often than the other groups after they were shown the correct argument.

Mercier and Sperber (2017) draw the following conclusion regarding the adaptivity of our biased reasoning: "If we take an interactionist perspective, the traits of argument production typically seen as flaws become elegant ways to divide cognitive labor. The most difficult task, finding good reasons, is made easier by the myside bias and by sensible laziness. The myside bias makes reasoners focus on just one side of the issue rather than having to figure out on their own how

${ }^{37}$ Contrary to that, if participants themselves reached the right conclusion and were then confronted with the argument that the answer is "Cannot be determined" because we do not know whether Linda is married or not, no one changed their mind. 
to adopt everyone's perspective. Laziness lets reason stop looking for better reasons when it has found an acceptable one. The interlocutor, if not convinced, will look for a counterargument, helping the speaker produce more pointed reasons. By using bias and laziness to its advantage, the exchange of reasons offers an elegant, cost-effective way to solve a disagreement." (p. 236)

We see that the main take-away of the interactionist approach is that groups perform better in producing sound arguments than individuals. Several studies confirm this assumption. For example, Moshman and Geil (1998) let participant do the selection task that Wason (1966) used in his study. In line with the results of Wason, participants performed badly if they had to do so alone. Here, only $9 \%$ selected the correct response pattern. Meanwhile, if subjects solved the same problem in small groups of five to six peers, $75 \%$ found the right response pattern. This number is extraordinarily high. In comparison, only $27 \%$ of Harvard students selected the correct pattern (Cosmides, 1989). The authors conclude that (1) the structure of arguments that groups co-constructed was typically qualitatively more sophisticated than that generated by most individuals; and (2) the superior performance of the groups was because of collaborative reasoning rather than peer pressure or imitation. Therefore, it was not simply the most confident person who pushed through his argument, whereby confidence correlated with the quality of the argument. On the contrary, the extracts of the discussion reveal that arguments were put forward one after another. Besides, Trouche et al. (2014) also demonstrated that a single participant with the correct answer can sway the whole group even though that participant initially was less confident than the other group members. In the corresponding experiment, the authors compared the performance of individuals and groups regarding the Paul-Linda-Ryan problem presented above. As in case of Moshman and Geil (1998), groups were significantly more likely to find the right answer than individuals (63\% vs. $22 \%$ ).

Many other studies suggest that group discussion often improves reasoning performance. They examined the power of groups by means of laboratory experiments in a wide range of tasks, including inductive problems (Laughlin et al., 2002), deductive problems (Laughlin \& Ellis, 1986; Moshman \& Geil, 1998; Trouche et al., 2014), numerical estimations (Minson et al., 2011; Sniezek \& Henry, 1989), and various work-related problems (Blinder \& Morgan, 2005; Lombardelli et al., 2005; Michaelsen et al., 1989). Moreover, collaborative reasoning was also found to be effective in other contexts as for example work teams (Guzzo \& Dickson, 1996), scientific discussions (Dunbar, 1995; Mercier \& Heintz, 2014; Okada \& Simon, 1997), political discussions (Fishkin, 2009; Mercier \& Landemore, 2012), mock juries (Hastie et al., 1983), and forecasting group teams (Mellers et al., 2014; Rowe \& Wright, 1996). Ultimately, group discussion leads 
to similar improvements throughout development, starting with preschool children (Doise \& Mugny, 1984; Mercier, 2011b; Perret-Clermont, 1980; Slavin, 1995; Smith et al., 2009b) and in different cultures including small scale hunter-gatherer societies (Mercier, 2011a; Mercier et al., 2016; Castelain et al. 2016). ${ }^{38}$ These results are robust given some minimal conditions are fulfilled, such as providing a heterogeneous opinion pool (Sunstein, 2002) and allowing everyone to express their true opinions (Janis, 1982). (Mercier et al., 2015)

The apparent insight that reasoning mainly serves social functions, notably argumentation, and that collaborative reasoning is an effective method to gain better beliefs is actually not a new one (Cattaneo, 1864; Bos, 1937; Joubert, 1932; Shaw, 1932; cf. Billig, 1996; Landemore, 2012). However, it did not gain much attention in academia. This is because not all studies that investigated group performance came to the conclusion that groups improve beliefs. There are particularly three phenomena that seem to scrutinise the "belief improving power of groups": groupthink, group polarisation, and belief perseverance.

Let us begin with groupthink. In the 1960 s, psychologist Irving Janis started examining when and why small groups make poor decisions. For example, one of his objects of investigation was the disastrous attack on Cuba launched by the American government in 1961 (the so-called Bay of Pigs invasion). Later, President John F. Kennedy who with his team planned and executed the invasion asked himself: "How could we have been so stupid?" In hindsight, the group truly made blatant misjudgements and entirely ignored critical objections. By analysing this case, Janis (1972) inferred that Kennedy and his team suffered groupthink. He defines groupthink as "a mode of thinking that people engage in when they are deeply involved in a cohesive ingroup, when the members' strivings for unanimity override their motivation to realistically appraise alternative courses of action" (p. 9). ${ }^{39}$ So, in a situation where members of a (cohesive) group fall into a state of groupthink, they try to minimise conflict so as to reach a consensus. Thereby, group members stop criticising each other's suggestions and fail to consider other alternatives. Typically, this produces an illusion of invulnerability, whereby the group overestimates their own abilities while underestimating those of the outgroup. The consequence of this are poor group decisions that are in fact

\footnotetext{
38 These cross-cultural findings are very important because they implicate that collaborative reasoning is not a cultural trait (e.g. learned in school) but a universal trait that evolved during the course of evolution (for a closer examination see Mercier and Sperber (2017), chapter 16). So, collaborative reasoning should comprise a fitness advantage.

${ }^{39}$ It has to be emphasised that high group cohesiveness is only one of three possible antecedents of groupthink. The other two are structural faults and the situational context. Yet, high group cohesiveness is the most important antecedent for Janis.
} 
poorer than the average decision of all group members given they had reached the decisions individually (Janis, 1982).

The second phenomenon that scrutinises the power of collaborative reasoning is called group polarisation. Group polarisation involves the tendency for a group to make decisions that are more extreme in the same direction as the original inclination of its members (Myers \& Lamm, 1976). Moreover, "deliberation tends to move groups, and the individuals who compose them, toward a more extreme point in the direction indicated by their own predeliberation judgments". (Sunstein, 2002, p. 175). Group polarisation was first discovered by James Stoner. Stoner (1961) gave participants a decision dilemma. In a chess tournament, a rather low-ranked chess player has to play against the top-favoured man. During the course of his play, he notes that he could now play a deceptive but risky move. If it works, he should quickly win. Yet, if his opponent reads it, he almost certainly loses. The participants had to individually indicate how high the chances that his move is successful should at least be in order that they would advise the chess player to do it. Then, they were put in groups where they had to solve the same problem and discuss it until they agreed on an answer. Stoner found that groups were typically riskier than their average individual members. However, he and other researchers that examined this so-called risky shift thought that it is a characteristic of risk itself and not of the procedure in general. Only a few years later, this got revised. For example, Teger \& Pruitt (1967) found that the mean initial response and the mean shift are highly correlated and thus given this mean initial response is rather cautious, groups become more cautious.

There are various empirical examples of group polarisation. A meta-analysis conducted by Isenberg (1986) found clear indications for the phenomenon and two main sources underneath it: social comparison and persuasive argumentation. Yet, on one hand, social comparison effects tended to be smaller. On the other hand, the research community disagrees about the importance of social comparison for group polarisation (Burnstein \& Vinokur, 1973, 1975, 1977). Thus, we exclusively focus on persuasive argumentation (Burnstein 1982; Vinokur and Burnstein 1978). ${ }^{40}$ According to this theory, "an individual's choice or position on an issue

\footnotetext{
${ }^{40}$ For the interested reader, here is a short description of how social comparison theory affects group polarisation, written by Burnstein and Vinokur (1977): "Social comparison theory, in one version or another (see the review by Pruitt, 1971), assumes: (a) a preference for alternative $\mathrm{X}$ is more socially desirable than a preference for alternative $\mathrm{Y}$; (b) the person believes his own preference for $\mathrm{X}$ is at least as extreme as those of his peers (in Brown, 1965; Festinger, 1954; Jellison \& Riskind, 1970) or is too extreme to be acceptable (in Levinger \& Schneider, 1969; Pruitt, 1971); (c) upon learning this is untrue, he experiences distress (in the Brown, Festinger, and Jellison \& Riskind version) or relief (in the Levinger \& Schneider and Pruitt
} 
is a function of the number and persuasiveness of pro and con arguments that that person recalls from memory when formulating his or her own position" (Isenberg, 1986, p. 1145). Now, in a group discussion, individuals collect and contribute arguments for the various positions that are supported. The decisive question is which of these arguments are persuasive and therefore later recalled? Two main factors define the persuasiveness of an argument: its validity and its novelty (Isenberg 1986; Burnstein 1982). The validity of an argument implies how true and sound it is plus how well it fits to my pervious views. The novelty of an argument involves questions such as does the argument represent a new way of organising information? Does it propose new ideas? Does it increase my access to additional information that are stored in my memory (Vinokur \& Burnstein 1978)? The combination of perceived validity and perceived novelty of a certain argument will then determine how big its influence in causing a choice shift is. How does this lead to group polarisation? If a group homogenously has attitude $\mathrm{X}$, its members mainly come up with arguments why attitude $X$ is correct because they suffer a myside bias. In so doing, each group member probably hears novel reasons why attitude $\mathrm{X}$ is correct, which leads to an even higher persuasiveness of pro-attitude $\mathrm{X}$ arguments. As a result, the group members gradually strengthened each other's belief in the correctness of attitude $\mathrm{X}$ or, to put it differently, they polarised each other.

Belief perseverance is the third phenomenon which challenges the interactionist approach. The interactionist approach requires that humans acknowledge the superiority of certain arguments. We have already discussed ample evidence that confirms this. However, sometimes we also get obsessed by a wrong belief and are not able to acknowledge the superiority of certain arguments. The French criminalist Alphonse Bertillon provides a rather extreme example of this. During the Dreyfus affair, Bertillon rendered a graphological expert opinion which stated that Alfred Dreyfus wrote the for the conviction relevant letter and therefore was guilty. Bertillon did so even though he had no prior experience in graphology and there were significant differences between Dreyfus' handwriting and the handwriting on the letter. As more and more doubts were casted on whether Dreyfus truly wrote the relevant letter, Bertillon defended his belief vehemently. He also kept doing so after a person was found that had the exact same handwriting as the one on the letter and even after this person confessed that he wrote the letter. Finally,

version); (d) either affective state causes the person to take a more extreme position which results in a decrease in distress (e.g., because according to Jellison \& Riskind he no longer appears less able than others) or an increase in satisfaction (e.g., because according to Pruitt he freely vents what was formally suppressed).” (p. 318) 
a few weeks before Bertillon died (Alfred Dreyfus was already completely rehabilitated), he was offered a long-awaited medal. Yet, in order to get it he had to admit his mistake in the Dreyfus affair. Unsurprisingly, he rather died without the medal than acknowledging his fault (Mercier \& Sperber, 2017).

The psychological phenomenon which Bertillon fell victim to is called belief perseverance. It means that a belief is upheld although there is overwhelming evidence against it (Anderson, 2007). Various experiments have detected belief perseverance (Anderson, 1995; Anderson et al., 1980; Anderson \& Lindsay, 1998; Ross et al., 1975). Moreover, belief perseverance has substantial overlaps with the continued influence effect for which ample empirical evidence exists as well (e.g. Ecker et al., 2010, 2011; Johnson \& Seifert, 1998; Seifert, 2002; van Oostendorp, 1996; van Oostendorp \& Bonebakker, 1999; Wilkes \& Leatherbarrow, 1988; Wilkes \& Reynolds, 1999). Thus, while Trouche et al. (2014) found that humans acknowledge the superiority of certain arguments, there are plenty of studies which demonstrate the opposite. As a consequence, it is doubtful how much group discussion improves our beliefs because even if it leads to better arguments, there seems to be no guarantee that we acknowledge the superiority of them.

Now, does belief perseverance, groupthink, and group polarisation smash the power of collaborative reasoning and thereby the interactionist approach? First of all, while the existence of group polarisation has been confirmed in a metaanalysis and is accepted in the psychological community, groupthink is much more controversial. On one hand, only few empirical studies have been published about groupthink. These studies provided only partial support for Janis' concept of groupthink (Park, 1990; Aldag \& Fuller, 1993). On the other hand, in a meta-analysis, Mullen et al. (1994) could not find a correlation between group cohesiveness (groupthink's most important antecedent) and quality of decisionmaking. In fact, high group cohesiveness can also have positive consequences because it can lead to more communication, less tension, and reduced anxiety of group members to speak up. Moreover, Packer and Chasteen (2010) examined groupthink out of a social identity perspective. They hypothesised that compared to low-identifiers, group members that strongly identify with their group $(=$ high cohesiveness) are more likely to represent a dissent argument if they believe it improves the situation of the group. Their experiments confirmed this hypothesis. In conclusion, the empirical evidence regarding groupthink is not strong enough in order that the interactionist approach has to be abandoned.

As mentioned above, the empirical evidence for group polarisation and belief perseverance is substantially stronger. How can these phenomena be compatible with the interactionist approach? Mercier and Sperber (2017) argue that the first key to this question is not to exclusively analyse how reasoning works but to also 
consider when it is triggered. According to the authors, this trigger is "a clash of ideas with an interlocutor" (p. 248). Therefore, our cognitive reasoning mechanisms are not primarily designed to find the best arguments individually or in a like-minded group but to do so in a group that experiences dissent. As Mercier et al. (2015) state, the minimum requirements for successful collaborative reasoning are a heterogeneous opinion pool (Sunstein, 2002) and allowing everyone to express their true opinions (Janis, 1982). If these requirements are fulfilled, group discussion often improves reasoning performance. ${ }^{41}$ The second key to the question of how group polarisation is compatible with the interactionist approach is to look at the environment, more precisely at the changes of the environment. During the late Pleistocene, humans lived in middle-sized groups of approximately 37 people (Marlowe, 2005). Their daily interactions were characterised by recurring social interactions. Thus, the normal conditions for the use of reasoning in the interactionist approach are social and therefore dialogic. Given this environment changes, the benefits of our reasoning mechanisms, which evolved in an antecedent environment, might vanish.

Now, if we compare today's environment with that of 50'000 years ago, we find substantial differences. First, compared to the late Pleistocene, we live much more individualistically today. As a result, our reasoning is no longer primarily applied in dialogues but very often reduced to inner monologues. This per se is not a problem. However, it becomes one if solitary reasoning remains solitary because if this is the case, there is no one that challenges the lone reasoner. As a consequence, the reasoner becomes more and more sure of her beliefs. This is amplified by another circumstance. Before the printing press was invented and

\footnotetext{
${ }^{41}$ For example, the shared information bias is another apparent dysfunction of groups. It says that groups have a propensity to spend more time on discussing information, which is already known to all members, and less time on discussing information, which is solely known to some members. The bias was particularly explored concerning group work. In a meta-review, Reimer et al. (2010) conclude: "Groups discussed more shared than unshared information overall. However, the observed sampling advantage was smaller than expected. Groups attenuated the discussion bias in particular when they had to choose among a small number of decision alternatives and when they had less than 30 minutes discussion time." (p. 121) While the shared information bias does not per se have to lead to uninformed group decisions and certainly does not imply that individual decisions would have been more accurate than group decisions, it is still irritating. An advantage of a group precisely is the ability to gather unshared information because more information should ultimately lead to a more carefully considered and thus better decision. So, it seems that groups waste their potential of making a proper decision because they mainly focus on shared instead of unshared information. Yet, a study conducted by Schulz-Hardt et al. (2006) revealed that already minority dissent within a group significantly reduces the shared information bias. This is particularly true if dissent came from a proponent of the correct solution. Again, this confirms the interactionist approach.
} 
modern media arose, "people were typically made aware that somebody in their own group had opinions different from theirs thanks to interaction with that person. Finding out about difference of opinion and trying to resolve them commonly occurred through repeated exchanges of arguments that could be anticipated and mentally rehearsed." (Mercier \& Sperber, 2017, p. 249) So, while the media, books, and blogs might still challenge our arguments, they do not produce a dialogic interaction. ${ }^{42}$ For example, a newspaper article provides a counterargument to our position. Due to the myside bias, after reading it, we start to find arguments why the article is wrong. The problem is that our new arguments will not be challenged by the author of the article because she is not there. Thus, our counterarguments to the arguments of the article might be weak but since there is no one who contradicts us we are satisfied with them.

Second, since the widespread advent of books and even more important the internet, we are able to quickly find people that share our opinion, regardless of how absurd it is. For example, there are numerous videos on Youtube about why the Earth is flat or why chemtrails are used so as to reduce human population. Or there are various books which state that $9 / 11$ was an inside-job or that there are aliens who kidnap humans and examine them. On one hand, someone who holds such beliefs and therefore is constantly challenged by the mainstream feels reaffirmed when she realises that there are others who think so too. On the other hand, such communities provide the ideal breeding ground for group polarisation. Conspiracy theories in particular have the self-enforcing feature to declare every counterargument as a cover-up attempt and thereby further prove for the conspiracy. In the late Pleistocene, the stubborn persistence of such weak and uncommon arguments should have been almost impossible. This is because first, as mentioned several times, reasoning was primarily dialogic. Second, the internet has billions of users. Therefore, you most certainly find others that agree on the same weak and uncommon beliefs as you do. In all likelihood, this was not the case in hunter-gatherer groups of circa 37 people. In other words, thanks to the internet these outliers of every group, which 50'000 years ago used to be convinced (or silenced) by group members at one point, can now build their own community.

Third, compared to the late Pleistocene, we first encounter a lot more people today and second these people not seldomly have different cultural backgrounds. This makes collaborative reasoning more difficult because culture normally entails

\footnotetext{
42 This also applies to experiments, where participants were solely given disconfirming evidence but then were no longer challenged in their new arguments by the experimenters (e.g. Ross et al., 1975).
} 
unspoken and unquestioned basic assumptions that might collide if people of different cultures argue with each other. Yet, the arguers are not aware of the fact that their dissent simply is a product of their different socialisation. This problem hardly existed in hunter-gatherer societies because discussions typically arose within groups and thus reasoning was based on the same cultural basic assumptions.

In summary, our cognitive reasoning mechanisms sometimes appear to be flawed (cf. belief perseverance, group polarisation). However, these flaws seem to be the product of environmental changes: Unlike our ancestors, today we (1) often reason alone and not in a dialogic context; (2) always find others that support our weak arguments; and (3) argue with people that have substantially different unspoken basic assumptions due to their cultural background. Because of these changes our reasoning is distorted and its adaptivity questioned. But as the literature review of Mercier et al. (2015) demonstrates, if we look at situations where these changes are inexistent, the way we reason (including the myside bias and selective laziness) is no longer a bug but a feature.

\subsubsection{The Role of Social Identity in the Belief Formation Process}

The last three subchapters have shown that people do at least not always update their beliefs according to Bayes' law. In this final subchapter, we want to analyse whether these deviations from Bayes' law are influenced by social identity. The idea behind this is as follows: In a decision situation, a seemingly agent-neutral individual justifies his preference of characteristics $\mathrm{X}$ provided by the ingroup over characteristics $X$ provided by the outgroup through his beliefs and therefore statistical discrimination. However, in fact, he also has a taste for the ingroup which he hides behind his claim to be a mere statistical discriminator. Now, let us assume that his beliefs truly suggest a preference of characteristics X provided by the ingroup over characteristics $\mathrm{X}$ provided by the outgroup. Could it be possible that his whole belief formation process was (and still is) distorted by his social identity in such a way that it led to beliefs that tend to flatter the ingroup and decry the outgroup?

Let us start with a study conducted by Nyhan and Reifler (2010). The authors wanted to investigate whether disconfirming evidence would change people's beliefs. For that they implemented four experiments in which participants had to read mock news articles which contained a misleading claim from a politician. 
Over the course of the article, this claim was either corrected or not. ${ }^{43}$ Then, they had to indicate whether they agree with a statement that supported the misleading claim of the politician. The results reveal that among the statement's targeted ideological group the corrections often failed to diminish misperceptions. But not only that, there were numerous instances where the corrections even backfired and led to stronger agreement with the statement. Therefore, at least some of the participants that were part of the statement's targeted ideological group seem to have updated their beliefs in a non-Bayesian way. Otherwise, it is hardly explainable why the correction of the misleading claim did lead to stronger approval of it. Furthermore, this non-Bayesian updating process helped them to maintain / strengthen their ideology.

Flynn et al. (2017) call the process that underlies these findings directionally motivated reasoning. According to Kunda (1990), different goals can be activated when people process information, as for example accuracy goals (trying to process information as dispassionately as possible) or directional goals (trying to reach a desired conclusion). Now, in case of directionally motivated reasoning, people seek out information that reinforces their view and avoid information that contradicts it. This is also called selective exposure. Additionally, because of directionally motivated reasoning "people may engage in motivated processing of the information they receive. More specifically, studies show that people tend to accept and recall congenial factual information more frequently than uncongenial facts (Jerit \& Barabas, 2012; Kahan, Dawson, Peters, \& Slovic, n.d.); interpret facts in a belief-consistent manner (Gaines et al., 2007); rationalize to maintain consistency with other beliefs (Lauderdale, 2016); and counterargue corrective information (Nyhan \& Reifler, 2010)" (Flynn et al., 2017, p. 132). So, people's reasoning and, in this way, their belief formation process can be influenced by directional goals. This leads to the following question: Might one directional goal of motivated reasoning be upholding a positive social identity? If that were the case, social identity would affect our belief formation process.

Dvir-Gvirsman (2019) examined the connection between selective exposure and political social identity. Political social identity is based on the idea that people interpret politics as a matter of identity and are as divided along political lines as they are for example by race (Huddy et al., 2015; Iyengar \& Westwood, 2015). The author found that the strength of political identity predicted selective exposure: Participants that strongly identified with a political camp rather chose an ideologically consistent than an ideologically inconsistent article. Importantly, this

\footnotetext{
${ }^{43}$ For example, one such mock news article concerned the alleged weapons of mass destruction of Iraq.
} 
effect was still significant after controlling for participants ideological extremity and their strength of political beliefs. Other studies confirm the finding that party identification, as a salient social identity, leads individuals to seek like-minded news outlets (Garrett \& Stroud, 2014; Iyengar \& Hahn, 2009; Stroud, 2011).

What about social identities beside politics? The study of Appiah et al. (2013) analysed selective exposure in regard to ethnic identity. The authors wanted to find out whether positive or negative valence of a news story and the ethnicity of the character portrayed in the story would affect white or black readers' selection of a story. There are three main results: (1) Black participants were more likely to select and read positive and negative stories that involved their ethnic ingroup, whereby positive stories prevailed. (2) Black participants were more likely to select and read negative stories about their outgroup compared to positive ones. (3) Whites' story selection was not influenced by story valence or character ethnicity. So, again, social identity seems to have influenced the information gathering process, yet, only in case of black participants.

The authors interpret these results as follows: First, the fact that black participants preferred positive to negative news stories when they featured a black person but negative to positive news stories when they featured a white person demonstrates ingroup favouritism and outgroup derogation. Second, the circumstance that black participants generally read more negative articles about Blacks than about Whites might be due to perceived similarity to characters. As Weaver (2011) argued: "[A]udiences may be motivated to select content featuring samerace characters either because of a perception that such content will portray the ingroup in a positive way (social identity theory) or because of a simple preference for characters similar to themselves (social cognitive theory)." (p. 371) Third, one's ethnicity is significantly more salient and important for black than it is for white people (Phinney, 1992). This is because they are a low-status / minority group. In accordance with that, black participants identified themselves more strongly with their ethnic ingroup than white participants. In turn, people that highly identify with their ingroup are more likely to display ingroup favouritism and outgroup derogation (Lewis \& Sherman, 2010; Vanhoomissen \& Van Overwalle, 2010). That is why selective exposure was only present in case of black participants.

Knobloch-Weserwick \& Hastall (2010) found that identification with a certain age group can lead to selective exposure. In an online news magazine, individuals of 18 to 30 years old mainly focused on same-aged individuals and in so doing preferably read positive news about their ingroup. In contrast, 50 to 65 years old participants rather read negative news about young individuals than positive news about this outgroup or than negative news about their ingroup. Moreover, 
such exposure to negative news about younger individuals reinforced older recipients' self-esteem. The authors conclude that these findings are to a great extent compatible with a social identity approach to selective exposure. ${ }^{44}$

Lastly, Wojcieszak \& Garrett (2018) primed participants so as to raise the salience of national identity. First of all, this had the effect that immigrant opponents on one hand attributed more negative traits and perceived more social distance to immigrants and on the other hand reported greater ingroup favourability. Therefore, priming national identity promoted affective polarisation. Second, it led immigration opponents to select more pro-attitudinal news stories, meaning stories that portrayed immigration negatively, and to spend more time reading these than their counterparts who did not get primed. According to the authors, these two findings are connected. They infer that "among immigration opponents, salient national identity exacerbates affective polarization both directly and through seeking content reaffirming people's prior views" (p. 267).

We see that the evidence presented in this subchapter indicates that social identity affects our belief formation process: ${ }^{45}$ Our identification with a group changes our belief formation process in such a way that it enables us to uphold or even bolster the positivity of our social identity. As a consequence, the beliefs of an agent-relative statistical discriminator can be influenced by his tastes and, in this way, he might hide his tastes behind his beliefs. Now, the studies discussed in this subchapter mainly concentrated on selective exposure. Unfortunately, no study could be found that examined the connection between the interpretation of a statement and social identity. However, there might be an indication for this connection in the experiment of Nyhan and Reifler (2010). As previously mentioned, only among a misleading statement's targeted ideological group the corrections often failed to diminish misperceptions and sometimes even backfired. This could suggest that participants' political social identity influenced their interpretation of the correction. But of course, this hypothesis needs further proof.

To summarise the whole chapter, there is ample evidence that humans do at least not always update their beliefs according to Bayes' law: We mistake availability for probability; have distorted memories of former prior probabilities; are good (bad) at producing arguments that confirm / verify our (an opposite) position but rather bad (good) at producing counterarguments; and are more critical of

\footnotetext{
${ }^{44}$ Furthermore, they are not compatible with social cognitive theory and social comparison theory.

45 There is also an opposing study in which exposure to pro-U.S. messages could not be predicted by identification with the American nation (Knobloch-Westerwick et al., 2017). Nonetheless, the circumstance that at least in some situations social identity affects selective exposure seems to be hardly deniable.
} 
other people's arguments than of our own. Additionally, social identity can affect our belief formation process in such a way that it leads to beliefs that tend to flatter the ingroup and decry the outgroup.

\subsection{About the Beliefs We Learn}

The last two chapters revealed that humans seem to have inherent prior beliefs and that we do not (exclusively) update our beliefs by use of Bayes' law. Now, let us ignore these circumstances for a moment and ask what beliefs someone with agent-neutral preferences would learn that starts with uniform priors and updates them by use of Bayes' law (e.g. an algorithm). Under these conditions, the learned beliefs would completely depend on the decision-maker's environment. In our case, this environment is the Western society and within this society, we would learn various beliefs about systematic differences between groups (and use them for statistical discrimination). In many cases, these differences cannot be explained by means of biology (alone). ${ }^{46}$ For example, why are there comparatively few black students at Ivy League Schools (Ashkenas et al., 2017)? Why are foreigners more likely to be convicted for a crime than natives (at least in Switzerland) (Schmidli et al., 2016)? Why are women less likely to major in natural sciences than men (Banaji \& Greenwald, 2013)? Why are blonds said to be stupid (Greenwood \& Isbell, 2002)? And why did Jews comparatively often work in the banking sector (Foxman, 2010)? If there is no biological explanation for these group differences, their origins have to be societal.

This chapter examines how societal characteristics affect the group specific beliefs we learn and thus is connected to previous chapters where we analysed the role of culture and cultural norms. ${ }^{47}$ Its goal is not to give an in-depth analysis of this topic but a sense of how society produces and preserves group specific beliefs. The chapter has the following structure: We first look at how historical circumstances can produce group specific beliefs that hold on for centuries. Then, we investigate why such beliefs do not (or only slowly) vanish but are reproduced. Finally, we give a short introduction to social dominance theory which tries to integrate sociological and psychological approaches to discrimination. In

\footnotetext{
${ }^{46}$ For example, the fact that only women give birth to children would be a systematic difference between men and women that can be explained by means of biology.

${ }^{47}$ Importantly, such societal characteristics can refer to both the meso-level (family, peers, etc.) and macro-level (society, core culture, etc.). However, in this chapter we rather concentrate on the macro-level.
} 
so doing, it provides a comprehensive explanation for why societies create group inequalities although the groups are (more or less) biologically equal.

\subsubsection{The Importance of Historical Circumstances}

If we look at beliefs that are not based on pure biology, we realise that these beliefs exist because of a prior (and maybe still prevailing) historical context. A perfect example of this are the stereotypes that link Jews with greed, money, and banking. ${ }^{48}$ In the Middle Ages, Jews were banned from many professions. They mainly had to carry out socially inferior jobs as for example tax and rent collecting or moneylending. The latter was particularly reserved for Jews because Christians were forbidden to lend money for interest. ${ }^{49}$ In fact, back then such practice was called usury, which only later changed its meaning to charging excessive interest. Thus, the Christian dominated and Jew-unfriendly society of the Middle Ages pushed Jews into money-lending since Christians needed someone who did this sinful job. Of course, this did not benefit the popularity of Jews, led to tensions between Jewish creditors and Christian debtors, and fuelled negative stereotypes about Jews such as they are greedy and heartless (Foxman, 2010).

William Shakespeare's play "The Merchant of Venice", which he wrote at the end of the $16^{\text {th }}$ century, portrays such stereotypes. ${ }^{50}$ Here, a Jewish money-lender named Shylock is one of the main characters. Shylock is asked to lend money to the Christian merchant Antonio who used to treat him unfavourably. He does so without wanting any interest. However, if Antonio is not able to pay back until a certain day, Shylock may take a pound of his flesh. As it happens, Antonio fails to repay the debt. So, Shylock goes to court so as to demand his pound of Antonio's flesh. He even declines Antonio's offer to repay the debt twice. In the end, Shylock has to surrender due to a legal loophole and loses everything because he gets convicted of attempted murder. Whether Shakespeare wanted to express his potential antipathy towards Jews through the character of Shylock is disputed (Ambrosino, 2016). Nevertheless, anti-Semites used the play for their propaganda.

\footnotetext{
${ }^{48}$ In this field of study, researchers normally use the word stereotype and not group specific belief, which is why we also primarily use the former. Yet, as previously mentioned, the two can be used interchangeably.

${ }^{49}$ Money-lending was perceived as a sin. This is rooted in the Old Testament (Exodus 22:25, Deuteronomy 23:19-20, Leviticus 25:35-37 and Psalms 15:5). Moreover, the only time Jesus got furious was when a temple was misused by merchants and money changers.

${ }^{50}$ Yet, in the end, Shylock gives a speech on tolerance (Hath not a Jew eyes?) and in so doing at least today regains some sympathy by the audience.
} 
For example, the Nazis broadcasted it shortly after Crystal Night in 1938 (Shapiro, 1996). Additionally, Shylock has become a synonym for loan shark. So, regardless whether Shakespeare was anti-Judean or not and wanted to display his attitude in his play, "The Merchant of Venice" unambiguously reveals three things: (1) how badly Jews were treated in the Middle Ages; (2) how such a play can be instrumentalised for political purposes; and (3) how a certain stereotype can form the collective consciousness (Shylock $=$ loan shark).

As time went by, Jews established in the upcoming financial sector. Most notable is the Rothschild family who set up a large banking imperium in the $18^{\text {th }}$ and $19^{\text {th }}$ century but who were also victims of various anti-Semitic conspiracy theories. These conspiracy theories cumulated in the idea of Weltjudentum, which fuelled antisemitism in the first half of the $20^{\text {th }}$ century and ultimately resulted in the Holocaust (Friedländer, 2007; Foxman, 2010). ${ }^{51}$ Finally, these Jewish stereotypes that emerged hundreds of years ago consist until today. In 2013, the Anti-Defamation League (ADL) conducted a poll in the U.S. $15 \%$ agreed that Jews are more willing to use shady practices than others. $19 \%$ of respondents believed that Jews have too much power in the business world. And $14 \%$ indicated that Jews are not as honest as other business people.

The way history has formed our stereotypes of a group is observable in various other cases. For example, only until recently, Western women were massively oppressed by men. They often could not learn a proper profession, might not even have gone to school, had to become housewives, could not participate in politics, could be raped by their husband, could be made to quit their job by their husband, and so on. Unsurprisingly, such a patriarchal society produced gender stereotypes that are asymmetric in their positive value. Broverman et al. (1972) examined such stereotypes in a time when gender roles started to be challenged. Still, they found clear patterns. While men were described as active, adventurous, rational, decisive, autonomous, competitive, ambitious, aggressive, worldly, and confident, women were seen as emotional, empathic, cautious, passive, quiet, dependent, insecure, soft, assimilated, and harmonising. Admittedly, there were also male stereotypes which have a negative connotation such as lack of interpersonal sensitivity, warmth, and expressiveness. ${ }^{52}$ Moreover, not all female stereotypes

${ }^{51}$ Of course, this is a simplified explanation of how these negative Jewish stereotypes came about and ultimately resulted in the Holocaust. Yet, there is an undeniable connection between the role of Jews in the Middle Ages as money-lenders, their later dominance in the financial sector, the conspiracy theories this produced, and the increasing usage of Jews as scapegoats at the beginning of the $20^{\text {th }}$ century.

52 Yet, back then, these characteristics might also have been perceived as weaknesses that a true man should not display. 
had a negative value. Yet, overall, stereotypical male characteristics were more often perceived to be desirable than stereotypical female traits. The authors add that a large segment of society also accepted these stereotypes: "[C]ollege students portray the ideal woman as less competent than the ideal man, and mental health professionals tend to see mature healthy women as more submissive, less independent, etc., than either mature healthy men, or adults, sex unspecified." (p. 75)

These stereotypes seem to date back more than two thousand years. The bible says that God made a woman from the rib he had taken out of a man (Genesis 2:22), making a clear statement of who is superior. This gets emphasised via statements such as: "For man did not come from woman, but woman from man" (Corinthians 11:8) and "Neither was man created for women, but women for man" (Corinthians 11:9). Then, it is Eve who takes a fruit from the tree of knowledge and eats it (Genesis 3:6). This makes her responsible for the original sin. ${ }^{53}$ Finally, there are several passages which state that wives should submit to their husbands (e.g. Collosians 3:18, Ephesians 5:22-24, Corinthians 11:3). Now, this shall not imply that the bible is the origin of patriarchal societies. In contrast, probably, the bible emerged in a society that already was patriarchal. ${ }^{54}$ Yet, it legitimised the oppression of women through a divine world order. And since the Western world was massively influenced by Christianity, these biblical gender roles of men and women survived for centuries.

If the law, which was made by patriarchal men, predetermines how women should live, if a patriarchal religion specifies the role of women, and if, as a product of that, a patriarchal society also expects women to behave in this way, is it surprising that many of them do? How should women be independent if the law makes them dependent from men? How should women be less submissive if the bible tells them to bow down to men. And how should women become more active and challenge the dominance of men if society expects them to be passive and harmonising? Out of this perspective, it is even more remarkable that thanks to strong feminist activism and immense willpower, women (at least partly) freed themselves from these stereotypes in the last 150 years.

To summarise, the exact beliefs of an agent-neutral decision-maker are closely intertwined with the society within which he learns and thereby that society's historical circumstances. Jews were not dominant in the financial sector because they had a genetical predisposition for that but because Christian dominated society

\footnotetext{
53 To be fair, she then gives it to Adam who takes a bite as well. So, both behave sinfully.

54 Social dominance theory provides an explanation for this social hierarchy. We will discuss it in section 4.3.3.
} 
pushed them into these professions. The question we want to ask in the next chapter is why such stereotypes can still prevail after societal restrictions seem to have vanished.

\subsubsection{Self-Fulfilling Prophecies and Reproduction of Social Conditions}

As we said in the last chapter, in today's Western societies, women have liberated (or are still liberating) themselves from many prior stereotypes. One of these is the gender-science stereotype: Men are good in science / math, whereas women are not. A hundred years ago, having such a stereotype was obvious because women did hardly have the chance to study science in the first place. So, how should they be good at it? However, there no longer are educational barriers for women. As a matter of fact, for instance in Switzerland, there are more women than men that complete a Higher School Certificate, which is the door opener for universities, and also more women than men that study at a university (Dubach et al., 2017). Yet, if we look at mathematical majors such as natural sciences or engineering, there are still significantly less women than men. For example, at the ETH, which is a polytechnic university, only one in three students is female (Nowotny, 2015). Why is that the case?

In 2005, Harvard University's former president Larry Summers gave a controversial answer to this question. Among other reasons, he said that women might be underrepresented in math and sciences because of a genetic lack of ability (Goldenberg, 2005). ${ }^{55}$ This statement is problematic because even if women were at that moment worse in math than men, this would not count as evidence that the observable gender difference has a biological origin. In fact, there are clear indications which suggest a different inference. As Banaji and Greenwald (2013) write: "The preponderance of boys with high SAT math scores has gone from a 10.7:1 ratio favoring boys in the $1980 \mathrm{~s}$ to $2.8: 1$ in the $1990 \mathrm{~s}$. In other words, the ratio favoring boys was nearly four times as large a mere decade earlier. Such a rapid closing of the gap between groups that used to be strikingly different should be surprising to those who favor a largely genetic explanation for gender differences in math ability, because genetically based differences cannot be reduced so dramatically in such a short period of time." (p. 121) Similarly, within 25 years,

${ }^{55}$ Due to this statement, Summers later resigned as president. 
the percentage of female ETH students rose from $18 \%$ to $33 \%$ (Riegelnig, 2012). Thus, apparently, the gender-science stereotype seems to get overridden, yet, this process takes time.

One major reason why the effects of such a stereotype do not immediately vanish after it is no longer officially endorsed is as follows: We might explicitly abandon such stereotypes but they continue to exist implicitly. Nosek et al. (2002) found that the stronger women's gender-science stereotype was in an IAT, which measures implicit associations, the less likely they preferred math or science. Moreover, the IAT score could also be used as a significant predictor for women's SAT math performance. Now, it might be objected that women hold such implicit stereotypes because they also hold them explicitly. Yet, there is ample evidence that not explicit but implicit stereotypes predict women's attitude towards math best. For example, Nosek and Smyth (2011) again found that, in case of women, stronger implicit gender-science stereotypes predicted worse math achievement, greater negativity toward math, weaker self-ascribed ability, and less participation. Importantly, these "implicit stereotypes had greater predictive validity than explicit stereotypes". (p. 1125) Another study conducted by Nosek et al. (2009) is even more intriguing. The authors analysed more than half a million genderscience IATs completed by citizens of 34 countries and reached the following three conclusions: (1) The level of a nation's implicit gender-science stereotype predicted nation-level sex differences in 8th-grade mathematics and science achievement. (2) Regarding this achievement gap, explicit stereotypes did not provide additional predictive validity. (3) "[I]mplicit stereotypes and sex differences in science participation and performance are mutually reinforcing, contributing to the persistent gender gap in science engagement" (p. 10593).

Another phenomenon that reveals how stereotypes can affect behaviour is called the stereotype threat. It was first discovered by Steele and Aronson (1995) who examined intellectual test performance of African Americans. Here, black participants performed worse if they thought that a test was diagnostic of ability or if a black stereotype (black people are less intellectual) was made salient before the test. Similarly, Spencer et al. (1999) studied whether women performed differently in a math test if the test was either described as "producing gender differences" or as "not producing gender differences". In line with Steele and Aronson (1995), they performed worse in the former condition. The explanation for these results is that the abovementioned conditions made a negative stereotype salient which disrupts performance because its holders become anxious about confirming the 
stereotype ${ }^{56}$ Now, these stereotypes do not have to be held explicitly. Galdi et al. (2014) examined stereotype threat among six-year-old children. Among these children, they found no indication that either boys or girls explicitly endorsed or were even aware of the gender-science stereotype. Yet, girls displayed automatic associations consistent with that stereotype. Furthermore, compared to a stereotype inconsistent condition, girls' math performance was significantly worse in a stereotype consistent condition. The decrease in performance was mediated by automatic associations. Ultimately, Kiefer and Sekaquaptewa (2007) suggest that if an implicit gender-science stereotype is strongly pronounced, no stereotypic cues are needed to create a stereotype threat. Here, stereotypes are chronically accessible and thus their impact ubiquitous.

The problem is that eventually implicit stereotypes can lead to a self-fulfilling prophecy (Merton, 1948). If a girl grows up in an environment that implicitly (and explicitly) portrays a gender-science stereotype, she might adopt it and actually perform worse in math, which confirms her implicit stereotype. Later, she might get aware of the stereotype and explicitly affirm it (at least in her case) due to her poor math performances. As a consequence, she plays along with the stereotype and rather studies languages or literature than math. And even if she never holds the stereotype explicitly, she might still be more interested in non-math subjects because she performs comparatively poorly in math which lessens motivation for it. On an aggregated level, this process maintains an implicit (and explicit) stereotype. Therefore, it is little surprising that it takes time until such a selffulfilling prophecy is broken and thereby the gender-science stereotype overcome. But as the rising number of female science students reveals, our society seems to be on the way to get there.

Yet, it is not always a stereotype alone that leads to a self-fulfilling prophecy and, in this way, keeps the stereotype alive. Often it is also a question of socioeconomic status (SES). For example, let us consider African Americans. The fact that African Americans are a disadvantaged group in the U.S. is again due to historical circumstances. The European American population, which dominated the U.S., used to enslave black people and continued to treat them unfavourably after they were liberated. Today, mistreatments of African Americans that are legitimised by the law have become rare. ${ }^{57}$ So, it seems like there are equal

\footnotetext{
56 The same phenomenon also exists in an exactly vice versa version. Here, it is called stereotype boost and implies that a group performs better after a positive stereotype was made salient (Shih et al., 1999, 2002).

57 There are still laws that mistreat African Americans (and other minorities). For example, in 2010, Arizona introduced a law (SB 1070) that particularly disadvantaged non-white people (Nill, 2011).
} 
opportunities for everyone now. However, the oppressed history of African Americans still impacts their momentary opportunities. For instance, $80 \%$ of students at Ivy League Schools are part of the richest fifth of U.S. society. The richest $2 \%$ represent even $20 \%$ of students (Hartman, 2006). Now, it might be objected that rich people are also more intelligent (that is why they are rich). Their offspring then inheres this intelligence which in turn is why they are overrepresented at elite universities. Yet, first of all, studying at such universities is expensive and even if a student might be qualified to study there, families with a low SES might not afford it. Second, there is an interaction between SES and intelligence. Turkheimer et al. (2003) analysed a sample of 7-year-old twins, who grew up in families with different SES. The authors detect that the influence of genes and the environment on intelligence is not linear across different levels of SES. Their models suggest that "in impoverished families, $60 \%$ of the variance in IQ is accounted for by the shared environment, and the contribution of genes is close to zero; in affluent families, the result is almost exactly the reverse." (p. 623) This means that a child in a low SES family could have the genes for high intelligence, yet, never fully expresses them because of her unstimulating environment. ${ }^{58}$ Consequently, while in theory there is equal opportunities, in reality, your SES predetermines them to a substantial degree.

So, negative African American stereotypes maintain due to African Americans' historically disadvantageous starting position and the consequent difficulty to catch up with European Americans. The situation is comparable with the board game monopoly that has the following rules: Player A gets $\$ 10$ '000, player B $\$ 2$ '000. Then, for the first 15 minutes, player $A$ has to pay half the price for all objects, whereas player B has to pay double the price. Moreover, the number player A dices gets doubled if she wants to. In contrast, the number player B dices gets always halved. After the first 15 minutes, both players play with the same rules. Unsurprisingly, even though player A has lost her privileges, she benefited so much from prior conditions that player B can hardly catch up. ${ }^{59}$ Likewise, it is difficult for African Americans to disprove the negative stereotypes about them if they live in a societal system that constantly reproduces the conditions that led to these stereotypes.

\footnotetext{
${ }^{58}$ For example, there are no books at home, parents do not express themselves eloquently, there is no discussion culture at the dinner table, the kids in the neighbourhood abhor school and insult students who like to learn as nerds, etc.

59 This is exactly where affirmative action wants to draw on. Through giving an advantage to certain groups, it wants to compensate the historical disadvantageous that these groups had to suffer and which still affect their momentary situation.
} 
In conclusion, stereotypes maintain because on one hand even if we explicitly abandon them, they can continue to exist implicitly, and in this way, still affect our behaviour. This can lead to self-fulfilling prophecies. On the other hand, today's societies are the product of past societies. If these past societies officially mistreated a certain group, it is possible that this circumstance still affects that group. This is because after the official mistreatment ended, the disadvantaged group started with such a backlog that they could not catch up yet. Thus, although theoretically all groups have equal opportunities, "initially" mistreated groups have a worse starting position and thereby much more obstacles on the way to the top. As a result, social conditions and stereotypes get reproduced.

\subsubsection{On the Structure of Society}

So far, we examined how historical circumstances influence the manifestation of stereotypes and why such stereotypes are difficult to overcome. Moreover, we saw that negative stereotypes were often applied on oppressed groups. This leads to the following question: Why are societies structured in a way that they generate dominant and oppressed groups in the first place? In this last chapter, we try to outline a brief answer to this question. In so doing, we discuss a theory that combines various psychological and sociological concepts, namely social dominance theory. The particularity of this theory is that it not only examines how individuals behave in a group context but also considers the societal structures the aggregated individual behaviour creates. In turn, these societal structures again affect individual behaviour.

The theory was developed by Sidanius and Pratto (2001) and begins with a basic observation that also inheres the question posed above: "[A]ll human societies tend to be structured as systems of group-based social hierarchies. At the very minimum, this hierarchical social structure consists of one or a small number of dominant and hegemonic groups at the top and one or a number of subordinate groups at the bottom." (p. 31) Here, the dominance of a group is characterised by a disproportionately large share of positive social value, which can be expressed in various ways as for example political authority and power, wealth, high social status, good and plentiful food, splendid homes, or the best available health care. Meanwhile, the subordinate group possesses a disproportionately large share of negative social value. Manifestations of this are low social status and power, relatively poor health care, high-risk and low-status occupations, poor food, severe negative sanctions (prison and death sentences), or modest if not miserable homes. 
These group-based social hierarchies consist of three distinct stratification systems ${ }^{60}$ : (a) an age system, where adults and middle-aged people dominate children and younger adults; (b) a gender system, where males dominate females; and (c) an arbitrary-set system. This last system can include all types of socially constructed and highly salient social categories as for example clan, estate, ethnicity, nation, caste, race, social class, regional grouping, religious sect, and so on. Again, within these social categories there is a group (e.g. white people) that has disproportionate social power over other groups (e.g. black people). As can be seen, these three systems differ regarding their fixedness. While we all at one point become adults if we live long enough and thereby join the high-status group, this does not apply to the gender system. If someone is born female she stays female her entire life and consequently never joins the high-status group. ${ }^{61}$ The arbitrary system is somewhere between. Certain social categories are very fixed such as skin colour. Others are more permeable such as social class. Yet, as the name implies, the definition of arbitrary systems is arbitrary. For instance, at which point a person is no longer considered to be white but black is randomly defined. ${ }^{62}$

The arbitrary system has two other characteristics. (1) While there is violence, brutality, and oppression in all three systems, typically, the most brutal oppression occurs in the arbitrary system. A demonstration of this circumstance provides the ever-present phenomenon of genocide. Of course, in the Western world, the most prominent genocide is the Holocaust. But there were many others too. For example, in the last fifty years, there was among others the Cambodian genocide, the East Timor genocide, the Kurdish genocide, the Isaaq genocide, the Bosnian genocide, the Rwandan genocide, and the genocide of Yazidis by ISIL. Furthermore, according to Genocide Watch (2018), there were five genocides occurring in 2018: in Syria, in Sudan, in the Democratic Republic of the Congo, in Ethiopia, and in Myanmar.

(2) The arbitrary system is generally not found among small hunter-gatherer societies (Lenski, 1984). Indeed, such societies might have social roles in form of a headman and / or a shaman that inhere a certain dominance. Yet, these roles

\footnotetext{
${ }^{60}$ A system of social stratification divides society into distinct groups with different statuses. For example, slavery was a system of social stratification, which divided society into those that are free (high status) and those that are enslaved (low status). Another example is socioeconomic status, which typically divides society into upper class (highest status), middle class, and lower class (lowest status).

61 At least, that was true for almost all of human history.

${ }^{62}$ For example, the U.S. used to have the one-drop rule, where already one black ancestor determined you as black.
} 
are normally assigned to those who prove to have the necessary individual skills. Thus, the hierarchies that follow from these social roles tend not to be transgenerational. In contrast, the age system and the gender system are also prevalent in hunter-gatherer societies. In case of the age system, Sidanius and Pratto (2001) do not explicate why this is true. But to be fair, such a statement is also not controversial. This is different regarding the gender system. Here, the authors write: "In both hunter-gatherer and early agricultural societies, while women contributed substantially to the subsistence of the group by frequently collecting and controlling the essentials for survival, there is no known society in which women, as a group, have had control over the political life of the community, the community's interaction with outgroups, or the technology and practice of warfare, which is arguably the ultimate arbiter of political power. ... Although there are several known examples of matrilineal societies (i.e., where descent is traced through the family of the mother), matrilocal or uxorilocal societies (i.e., where newly married couples reside with the wife's kin), and societies in which women have near economic parity with men, there are no known examples of matriarchal societies (i.e., where women, as a group, control the political and military authority within the society)." (p. 36)

If hunter-gatherer societies were mainly structured by only two stratification systems but modern societies have a strong third one, we have to ask the following question: What is it that promoted the emergence and / or strengthening of the arbitrary system? According to Sidanius and Pratto, the answer is economic surplus, more precisely the lack of economic surplus in hunter-gatherer societies and its existence in modern societies. Hunter-gatherer societies had no technologies to produce or store food that permitted long-term storage. Moreover, since hunter-gatherer societies usually are nomadic, people cannot accumulate large numbers of nonedible forms of economic surplus such as weapons, armaments, or animal skins. Because of that the development of highly specialised social roles, as for example professional police, armies, and other bureaucracies that enable the formation of political authority is hardly possible. Contrary to that, in modern societies, there is no necessity that all adults devote most of their time to food procurement and survival. Consequently, certain males are able to specialise in the arts of coercion (e.g. warlordism, policing) or intellectual / spiritual sophistry. In turn, "these specialists are used by political elites to establish and enforce expropriative economic and social relationships with other members of the society. Once these role specializations and expropriative relationships are in place, arbitrary-set, group-based hierarchies then emerge." (p. 35)

These observations bring us to the three primary assumptions of social dominance theory: “(1) While age- and gender-based hierarchies will tend to exist 
within all social systems, arbitrary-set systems of social hierarchy will invariably emerge within social systems producing sustainable economic surplus. ... (2) Most forms of group conflict and oppression (e.g., racism, ethnocentrism, sexism, nationalism, classism, regionalism) can be regarded as different manifestations of the same basic human predisposition to form group-based social hierarchies. ... (3) Human social systems are subject to the counterbalancing influences of hierarchy-enhancing forces, producing and maintaining ever higher levels of group-based social inequality, and hierarchy-attenuating forces, producing greater levels of group-based social equality." (p. 38)

Especially the second assumption reveals the difference between social identity theory, on which we mainly focused in this dissertation, and social dominance theory. While the former primarily looks at ingroup favouritism from an individual perspective, the latter does consider the societal implications of that as well. So, given no group-based social hierarchy can be identified in a society (or simply between two groups), social dominance theory has little to explain about the existence of ingroup favouritism. ${ }^{63}$ Here, it references to other theories such as social identity theory. Yet, as the above paragraphs demonstrate, this is seldomly the case outside the laboratory. Thus, when we look at actual attitudes and stereotypes, social dominance theory enables additional explanatory power. This is because it does not only consider individual processes but also takes the societal environment into account, namely a group-based social hierarchy, within which these processes take place.

As the third assumption of social dominance theory suggests, societies are exposed to two counterbalancing forces: hierarchy-enhancing and hierarchyattenuating ones. Good examples of the latter force are the various human rights movements of for instance women, blacks, or homosexuals that appeared in the last 70 years. In contrast, the biblical verses mentioned in section 4.3.1, which state that women are inferior to men, are examples of the hierarchy-enhancing force. Now, such stories as these biblical verses play an important role in social dominance theory and are called legitimising myths. They consist of attitudes, values, beliefs, and ideologies that justify the social practices which distribute social value within the social system. Hierarchy-enhancing legitimising myths are typically set up and spread by the dominant group and thereby can serve as a disguise for their tastes: Dominant and oppressed groups are treated differently due to the "myth's content" and not the tastes of the dominant group. But of course, these two probably are very much intertwined since social identity can affect our

${ }^{63}$ For example, this is the case in minimal group experiments. 
belief formation process (cf. section 4.2.4) and thus also what a legitimising myth contains.

Such myths can be straightforward as for example the misogynist biblical verses. Other instances provide anti-Jewish stories during the Middle Ages which stated that Jews poisoned wells and therefore are the causer of the plague or that Jews ritually kill Christian children (Cohn, 2007). Yet, legitimising myths can also be subtle. Let us consider the idiom "from rags to riches" which became an allegory of the American dream. It implies that regardless of your socioeconomic background you can achieve anything if you really want to. Consequently, if you do not make it from rags to riches, it is not because of the system but because of you. This leads to internal attributions of the misfortunes of those with a low socioeconomic status, which in turn prevents their desire to change the system because that would require an external attribution.

Despite the fact that social dominance theory has more aspects, this is where our outline of it ends. We do so out of three reasons. First, although Sidanius and Pratto position their theory as an exclusive explanation for ingroup favouritism, the parts discussed so far can also be conceived as a transmission of social identity theory on the structure of society. If groups compete against each other for status, and at least one group wins this competition, it is of little surprise that this produces hierarchy-based social systems. In such a society, the high-status group then wants to maintain its status and in so doing uses legitimising myths or enshrines its power in institutions. Thus, what social dominance theory in their second assumption calls the basic human predisposition to form group-based social hierarchies could simply be the societal consequence of social identity theory.

Second, while social dominance theory got quite some academic attention in the 1990 s and early 2000 s (e.g. Sidanus et al., 1992, 2004; Pratto, 1999; Pratto et al., 2006) ${ }^{64}$, the theory more or less disappeared in the last ten years. Instead, researchers rather focused on social dominance orientation. ${ }^{65}$ Social dominance orientation is a personality psychological scale that indicates a person's attitudes toward hierarchies and beliefs about whether one's own group should dominate other groups (Morrison \& Ybarra, 2007). Now, the absence of social dominance theory in the momentary academic discourse does not per se imply that the theory is incorrect. Yet, it suggests that social dominance theory did not prevail against

\footnotetext{
${ }^{64}$ Admittedly, many (if not most) papers about social dominance theory either have Sidanius or Pratto (or both) as author or co-author.

${ }^{65}$ For example, while the encyclopedia of social psychology of Baumeister and Vohs (2007), which describes more than 600 social psychological theories / phenomena, has a chapter for social dominance orientation, it does not have one for social dominance theory.
} 
social identity theory. In fact, social dominance theory was massively criticised by social identity theorists (e.g. Schmitt et al., 2003; Wilson \& Lui, 2003) and even declared as having been falsified (Turner et al., 2003).

Third, section 3.3 revealed ultimate explanations for social identity theory. Depersonalisation can be adaptive if there is a group selection mechanism or the group is rather small and mainly kin-based. Moreover, parochial altruism also provides an explanation for why humans not only display ingroup love but sometimes also outgroup derogation. Contrary to that, Sidanius and Pratto (2001) base their whole ultimate explanation of social dominance theory on the difference between male and female reproductive strategies. ${ }^{66}$ Indeed, this approach might persuasively explain the gender differences in social dominance orientation. However, it does not give a convincing answer for the question of why humans' far-reaching altruistic behaviour and ingroup favouritism should be adaptive. Turner et al. (2003) even describe the evolutionary basis of the social dominance drive as largely fantasy.

So, why bother about social dominance theory at all? Despite its weaknesses, Sidanius and Pratto's analysis undeniably demonstrates that the structure of societies is determined by hierarchy-based social systems. Furthermore, so as to keep their status and privileges, the ones that are at the top generally want to keep those that are at the bottom at the bottom. In so doing, the superior group makes selfbeneficial laws and spreads legitimising myths that function as justifications for the existing social hierarchy. The examples presented in section 4.3.1 and 4.3.2 perfectly demonstrate that. Ultimately, this can lead to a seemingly strange phenomenon, namely outgroup favouritism. Yet, such behaviour is not in conflict with social identity theory. Tajfel and Turner (1979) write: "[S]ubordinate groups ... [can] internalize a wider social evaluation of themselves as 'inferior' or 'second class', and this consensual inferiority is reproduced as relative self-derogation." (p. 37) According to the authors, this occurs if the following requirements are met: "[W]here social-structural differences in the distribution of resources have been institutionalized, legitimized, and justified through a consensually accepted status system (or at least a status system that is sufficiently firm and pervasive to prevent the creation of cognitive alternatives to it), the result has been less and not more ethnocentrism in the different status groups." (ebd.) In other words, social identity theory includes the possibility that low-status groups display outgroup favouritism when intergroup status is stable and legitimate (Turner \& Reynolds,

\footnotetext{
${ }^{66}$ Since women bear a child and as a result carry all the costs of pregnancy they have to choose their partner wisely. In contrast, for men, the costs of impregnation are marginal, which is why they are less picky.
} 
2001; Rubin \& Hewstone, 2004). Now, social institutions (e.g. the law) that are beneficial for the superior group and according legitimising myths precisely promote such stable and legitimate intergroup statuses. This is why inferior groups not always take collective action and sometimes even contribute to the maintenance of the status quo and thereby their own inferiority (Jost et al., 2004). Yet, as soon as the legitimising myths start to be questioned and the low-status group recognises the chance of change (as it often happened in the last decades), outgroup favouritism turns into ingroup favouritism. ${ }^{67}$ Again, in the words of Tajfel and Turner (1979): "[C]onsensual inferiority will be rejected most rapidly when the situation is perceived as both unstable and illegitimate." (p. 45)

To summarise the whole section 4.3, the precise beliefs about (and attitudes towards) one's ingroup and outgroups highly depend on the society within which an individual lives. While there certainly are beliefs that ground on biological facts, many if not most are socially construed or massively socially exaggerated. Such social facts influence the behaviour of people and can lead to self-fulfilling prophecies. Due to that they are hard to overcome. This is particularly true since those who dominate a society usually have no interest in overcoming the negative stereotypes of inferior groups because that would attack their own superiority. Furthermore, there are societal constellations where the low-status group does not favour itself but the high-status group and in this way helps to preserve the actually disadvantageous status quo. So, when we examine what beliefs people (or algorithms) learn, it is essential to analyse the learning environment of these people (or algorithms) as well. Because given this environment is co-shaped by taste-based discriminators (which is usually the case), the beliefs of an agentneutral Bayesian decision-maker will be affected by them.

\footnotetext{
${ }^{67}$ It is important to notice that whether intergroup status is stable or instable / legitimate or illegitimate is a subjective evaluation. Thus, some of a group might perceive an intergroup status as instable and illegitimate and therefore display ingroup favouritism in form of social competition, whereas others perceive it as stable and legitimate and thus show outgroup favouritism in form of defending the status quo.
} 
Open Access This chapter is licensed under the terms of the Creative Commons Attribution 4.0 International License (http://creativecommons.org/licenses/by/4.0/), which permits use, sharing, adaptation, distribution and reproduction in any medium or format, as long as you give appropriate credit to the original author(s) and the source, provide a link to the Creative Commons license and indicate if changes were made.

The images or other third party material in this chapter are included in the chapter's Creative Commons license, unless indicated otherwise in a credit line to the material. If material is not included in the chapter's Creative Commons license and your intended use is not permitted by statutory regulation or exceeds the permitted use, you will need to obtain permission directly from the copyright holder.

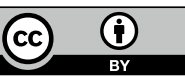

